\title{
A Nonstandard Higher-Order Variational Model for Speckle Noise Removal and Thin-Structure Detection
}

\author{
Hamdi Houichet ${ }^{1}$, Anis Theljani ${ }^{2}$, Badreddine Rjaibi ${ }^{1}$ \\ and Maher Moakher ${ }^{1, *}$ \\ ${ }^{1}$ Laboratory for Mathematical and Numerical Modeling in Engineering Science, \\ University of Tunis El Manar, National Engineering School at Tunis, B.P. 37, \\ 1002 Tunis-Belvédère, Tunisia. \\ ${ }^{2}$ Liverpool Centre for Mathematics in Healthcare, Department of Mathematical \\ Sciences, University of Liverpool, Liverpool, UK.
}

Received February 6, 2019; Accepted August 1, 2019

\begin{abstract}
We propose a multiscale approach for a nonstandard higher-order PDE based on the $p(\cdot)$-Kirchhoff energy. We first use the topological gradient approach for a semi-linear case in order to detect important objects of the image. We consider a fully nonlinear $p(\cdot)$-Kirchhoff equation with variable-exponent functions that are chosen adaptively based on the map provided by the topological gradient in order to preserve important features of the image. Then, we consider the split Bregman method for the numerical implementation of the proposed model. We compare our model with other classical variational approaches such as the TVL and bi-harmonic restoration models. Finally, we present some numerical results to illustrate the effectiveness of our approach.
\end{abstract}

AMS subject classifications: 65M32, 68U10, 35G25

Key words: Inverse problems, regularization procedures, $p(\cdot)$-Kirchhoff, topological gradient, split Bregman.

\section{Introduction}

Image restoration is a fundamental task in image processing and it arises in diverse fields like geophysics, optics, medical imaging, etc. In this work, we are interested in the restoration of images highly corrupted with multiplicative noise. Such a problem is a

${ }^{*}$ Corresponding author. Email addresses: hamdi.houichet@gmail .com (H. Houichet), thaljanianis@gmail . com (A. Theljani), badreddine.rjaibi@lamsin.rnu.tn (B. Rjaibi), maher.moakher@enit.utm.tn (M. Moakher) 
challenging task in various fields and particularly in ultrasound medical imaging. The reason is that ultrasound images are strongly influenced by the quality of data usually corrupted with Rayleigh-distributed multiplicative noise. The latter is called speckle noise $[40,41,44]$ and usually affects image analysis methods by making important features hard to detect. We aim at reconstructing an image $u: \Omega \rightarrow \mathbb{R}$ from an observed one $f: \Omega \subset \mathbb{R}^{2} \rightarrow \mathbb{R}$ which is degraded and contaminated by noise. The degradation model that we consider is the following:

$$
f=u+\eta \sqrt{u},
$$

where $\eta: \Omega \rightarrow \mathbb{R}$ is a positive function that follows the Rayleigh-distribution. The reconstruction problem based on model (1.1) is well known to be an ill-posed inverse problem and thus regularization techniques are needed. Generally, the regularization technique turns the reconstruction problem based on model (1.1) into a well-posed optimization one where the energy to be minimized is the sum of a regularization term (mostly a semi-norm of a functional space fixed a priori) and a data fitting term. The well-posed minimization problem has the following form:

$$
\min _{\{u>0 ; u \in \mathcal{H}\}}\left\{\mathcal{J}(u):=J(u)+\lambda \int_{\Omega}\left(\frac{f-u}{\sqrt{u}}\right)^{2} \mathrm{~d} x\right\} .
$$

The first part in the energy $\mathcal{J}(\cdot)$ is a regularization term, the second one is the fitting term, $\lambda$ is a positive weight which controls the trade-off between them and $\mathcal{H}$ is the space where the solution is sought.

Motivated by the advantages of the total variation, the authors in [40] proposed a convex variational model which consists in minimizing

$$
\min _{\{u>0 ; u \in \mathcal{H}\}}\left\{\mathcal{J}(u):=\int_{\Omega}|D u|+\lambda \int_{\Omega}\left(\frac{f-u}{\sqrt{u}}\right)^{2} \mathrm{~d} x\right\} .
$$

However, the total variation regularizer produces staircase effects in the restored images. In [58], the authors proposed the fitting term

$$
\int_{\Omega}\left(\frac{f}{\sqrt{u}} \log \frac{f}{u}-\frac{f}{\sqrt{u}}+\sqrt{u}\right) \mathrm{d} x
$$

which is derived from the Kullback-Leibler divergence, also known as the I-divergence [45]. After that, they used first- and second-order total variation as a regularization which yields the following model:

$$
\min _{u \in \mathcal{H}_{1}}\left\{\alpha \int_{\Omega}\left|D^{2} u\right| \mathrm{d} x+\beta \int_{\Omega}|D u| \mathrm{d} x+\lambda \int_{\Omega}\left(\frac{f}{\sqrt{u}} \log \frac{f}{u}-\frac{f}{\sqrt{u}}+\sqrt{u}\right) \mathrm{d} x\right\},
$$

where $\alpha, \beta$ and $\lambda$ are regularization parameters. Instead of solving the model (1.4), they in practice used the auxiliary variable $z=\log u$ and then solved:

$$
\min _{z \in \mathcal{H}_{2}}\left\{\alpha \int_{\Omega}\left|D^{2} z\right| \mathrm{d} x+\beta \int_{\Omega}|D z| \mathrm{d} x+\lambda \int_{\Omega}\left(f e^{-z / 2} \log \frac{f}{e^{z}}-f e^{-z / 2}+e^{z / 2}\right) \mathrm{d} x\right\} .
$$


The denoised image $u$ is then obtained by applying the exponential transformation to the denoised image $z$ in the logarithm domain. It is clear that there is no equivalence between the two models (1.4) and (1.5) as the regularization in the former is applied in the logarithmic domain. A better formulation would be the following constrained optimization problem:

$$
\begin{aligned}
& \min _{(u, z)} \alpha \int_{\Omega}\left|D^{2} u\right| \mathrm{d} x+\beta \int_{\Omega}|D u| \mathrm{d} x+\lambda \int_{\Omega}\left(f e^{-z / 2} \log \frac{f}{e^{z}}-f e^{-z / 2}+e^{z / 2}\right) \mathrm{d} x, \\
& \text { such that } z=\log u,
\end{aligned}
$$

which can be solved using the alternating direction method of multipliers or the augmented Lagrangian method.

The main issue in image denoising is how to choose the "best" regularization ter$\mathrm{m}$ which can selectively smooth a noisy image without losing significant features such as edges and thin structures. Various regularizers based on first- or/and second-order derivatives have been used $[11,13,17,50,53]$, yielding various linear and nonlinear diffusion PDEs. Usually, a linear diffusion PDE is more interesting and effective in homogeneous regions, whereas, a nonlinear diffusion PDE is more powerful in regions containing edges and details. Motivated by the effectiveness of both linear and nonlinear diffusion models, we consider in this work a new regularization functional which is compromise between them. More precisely, we consider the following regularizer:

$$
J^{p, q}(u):=\alpha \int_{\Omega} \frac{1}{p(x)}|\Delta u|^{p(x)} \mathrm{d} x+\beta \int_{\Omega} \frac{1}{q(x)}|\nabla u|^{q(x)} \mathrm{d} x,
$$

where the functions $p(\cdot)$ and $q(\cdot)$ are defined on $\Omega$ and satisfy $1<p(\cdot), q(\cdot) \leq 2$. Considering the above regularizer will lead to nonstandard PDEs with variable exponents $p($.$) and$ $q(\cdot)$. This kind of PDEs, especially for the $p$-Laplace equation, were considered in several works (see e.g. $[9,29,43]$ ). A lot of recent research studied partial differential equations and variational problems with $p(\cdot)$-growth conditions which arise in electro-rheological fluids [51], elasticity theory [61] and image processing [19,36, 42].

The smoothing proprieties of these nonstandard PDEs are driven by the variable exponents $p(\cdot)$ and $q(\cdot)$. The approach that we propose automatically balances between the $L^{1}$-Laplace (respectively $L^{1}$-gradient) and the $L^{2}$-Laplace (respectively $L^{2}$-gradient) regularization effects. Thus, by giving the variable exponents $p(\cdot)$ and $q(\cdot)$ the possibility to take values between 1 and 2 to slow diffusion (nonlinear) near edges, and enhance it (linear) in smooth regions. This choice avoids over smoothing and stair-casing artifact effects of $L^{2}$ - and $L^{1}$-regularization. However, the main question is how to choose the values of the exponents $p(\cdot)$ and $q(\cdot)$. A classical approach is to make an adaptive choice for $p(\cdot)$ and $q(\cdot)$. We first identify the important features using classical edge and thinstructure detector and then we vary the exponents $p(\cdot)$ and $q(\cdot)$, according to an edge map, in the restoration process by considering a fully nonlinear $p(\cdot)$-Kirchhoff model for $1<p(\cdot), q(\cdot) \leq 2$. For the edge and thin-structure detection task, we consider the topological gradient approach. The natural choice is to consider the nonlinear $p(\cdot)$-Kirchhoff 
energy. Unfortunately, it has not yet been studied thoroughly for $p(\cdot)$-biharmonic operators and we do not address it in this work. However, it was recently studied in $[6,22]$ for the biharmonic operator with very satisfactory result in the detection of fine features (the arteries, filaments, internal organs, etc.). We extend the result obtained in $[6,7,22]$ by considering the topological gradient for the particular case $p(\cdot) \equiv q(\cdot) \equiv 2$.

This paper is organized as follows. In Section 2, we fix notations and recall useful results for the generalized Lebesgue/Sobolev spaces. In Section 3, we prove, by standard variational techniques, the existence and uniqueness of the minimizer of the energy functional (1.2). In Section 4, we give the formula of the topological gradient for $p(\cdot) \equiv q(\cdot) \equiv 2$. In Section 5, we present the adaptive algorithm and we present the split-Bregman scheme for the restoration process. Finally, in Section 6, we treat some numerical examples to test the efficiency and robustness of the proposed model.

\section{Preliminaries and notations}

Before going further, we recall some useful definitions and results about the variableexponent generalized Lebesgue and Sobolev spaces $L^{p(\cdot)}(\Omega)$ and $W^{k, p(\cdot)}(\Omega)$. For more details, we refer the reader to $[21,24,25,27,28]$. For a bounded Lipschitz open set $\Omega \subset$ $\mathbb{R}^{N}$ with sufficiently smooth boundary $\partial \Omega$, we define the variable-exponent generalized Lebesgue space $L^{p(\cdot)}(\Omega)$ as follows:

$$
L^{p(\cdot)}(\Omega):=\left\{u: \Omega \rightarrow \mathbb{R} \text { measurable and } \int_{\Omega}|u(x)|^{p(x)} \mathrm{d} x<\infty\right\},
$$

where $p(\cdot) \in C(\bar{\Omega})$ be a measurable function, called variable exponent on $\Omega$ and satisfy the following condition:

$$
1<p^{-}:=\inf _{x \in \bar{\Omega}} p(x) \leq p(x) \leq p^{+}:=\sup _{x \in \bar{\Omega}} p(x) \leq 2 .
$$

Here $L^{p(\cdot)}(\Omega)$ is a normed linear space equipped with the Luxemburg norm [21]:

$$
\|u\|_{L^{p(\cdot)}}=\inf \left\{\varrho>0: \int_{\Omega}\left|\frac{u(x)}{\varrho}\right|^{p(x)} \mathrm{d} x \leq 1\right\} .
$$

Similarly, the Sobolev space with variable exponent $W^{k, p(\cdot)}(\Omega)$ is defined as:

$$
W^{k, p(\cdot)}(\Omega)=\left\{u \in L^{p(\cdot)}(\Omega): D^{\xi} u \in L^{p(\cdot)}(\Omega),|\xi| \leq k\right\},
$$

where $D^{\xi} u=\frac{\partial|\xi|}{\partial x_{1}^{\alpha_{1}} \partial x_{2}^{\xi_{2}} \ldots \partial x_{N}^{\xi_{N}}} u$ with $\xi=\left(\xi_{1}, \ldots, \xi_{N}\right) \in \mathbb{N}^{N}$ is a multi-index and $|\xi|=\sum_{i=1}^{N} \xi_{i}$. The space $W^{k, p(\cdot)}(\Omega)$, is equipped with the norm:

$$
\|u\|_{k, p(\cdot)}:=\sum_{|\xi| \leq k}\left\|D^{\tilde{\zeta}} u\right\|_{L^{p(\cdot)}}
$$


We recall that both $\left(L^{p(\cdot)}(\Omega),\|\cdot\|_{L^{p(\cdot)}}\right)$ and $\left(W^{k, p(\cdot)}(\Omega),\|\cdot\|_{k, p(\cdot)}\right)$ are separable, reflexive and uniformly convex Banach spaces [28].

Remark 2.1. We consider the following space $X=\left\{u \in W^{2, p(\cdot)}(\Omega)\left|\frac{\partial u}{\partial n}\right|_{\partial \Omega}=0\right\}$. The norm $\|u\|_{2, p(\cdot)}$ is equivalent to the following norm

$$
\|u\|_{X}=\inf \left\{\varrho>0: \int_{\Omega}\left|\frac{\Delta u(x)}{\varrho}\right|^{p(x)} \mathrm{d} x+\int_{\Omega}\left|\frac{u(x)}{\varrho}\right|^{p(x)} \mathrm{d} x \leq 1\right\},
$$

in the spaces $W^{2, p(\cdot)}(\Omega)$ and $X$ (see [10] for more details). Moreover, $\left(W^{2, p(\cdot)}(\Omega) ;\|\cdot\|_{X}\right)$ and $\left(X ;\|\cdot\|_{X}\right)$ are separable and reflexive Banach spaces. The norm $\|\cdot\|_{X}$ is equivalent to the following norm

$$
\|\Delta u\|_{L^{p(\cdot)}}+\|u\|_{L^{p(\cdot)}} \cdot
$$

Proposition 2.1. Let $p(x), r(x) \in C_{+}(\bar{\Omega})$.

a) If $p(x) \leq r(x)$ for $x \in \bar{\Omega}$, then $L^{r(x)}(\Omega) \hookrightarrow L^{p(x)}(\Omega)$ and the embedding is continuous.

b) Let $A$ be one the operators $I_{d}, \nabla$, and $\Delta$. Then, for $u \in W^{2, p(\cdot)}(\Omega)$, we have:

$$
\begin{gathered}
\|A u\|_{L^{p(\cdot)}}^{p-} \leq \int_{\Omega} \frac{1}{p(x)}|A u|^{p(x)} \mathrm{d} x \leq\|A u\|_{L^{p(\cdot)}}^{p+}, \text { if }\|A u\|_{L^{p(\cdot)}} \geq 1, \\
\|A u\|_{L^{p(\cdot)}}^{p+} \leq \int_{\Omega} \frac{1}{p(x)}|A u|^{p(x)} \mathrm{d} x \leq\|A u\|_{L^{p(\cdot)}}^{p-}, \text { if }\|A u\|_{L^{p(\cdot)}} \leq 1 .
\end{gathered}
$$

Proof. The proof of this proposition is similar to the proof of Theorem 1.3 in [28].

\section{Mathematical formulation of the problem}

In this paper, we discuss the image restoration problem, using model (1.2), based on the minimization of the following energy functional:

$$
\mathcal{J}(u)=\left\{J^{p, q}(u)+\lambda \int_{\Omega} W(u, f) \mathrm{d} x\right\},
$$

where $W$ is the continuous function defined by:

$$
W(s, z):=\left(\frac{z-s}{\sqrt{s}}\right)^{2}, \quad \forall s, z>0 .
$$

Moreover, the first derivative of $W$ with respect to $s$ is

$$
\frac{\partial W}{\partial s}(s, z)=(s-z) \phi(s) \quad \text { where } \phi(s)=\frac{(z+s)}{s^{2}} .
$$


It is easy to see that $\frac{\partial^{2} W}{\partial^{2} s}(s, z)=\frac{2 z^{2}}{s^{3}}>0$ and that $W(s, z)$ is a strictly convex function for $s>0[40]$.

Moreover, let $\underline{m}, \bar{M}>0$ such that $\underline{m} \leq z \leq \bar{M}$, the following inequality holds

$$
\frac{\partial W}{\partial s}(\underline{m}, z) \leq 0 \text { and } \frac{\partial W}{\partial s}(\bar{M}, z) \geq 0 .
$$

For brevity, we sometimes write $W(\cdot)$ for $W(\cdot, f)$ and $D_{u} W(\cdot, f)$ for $\frac{\partial W}{\partial s}(\cdot, f)$.

\subsection{Existence and uniqueness of solution}

We recall that if $\left(E,\|\cdot\|_{E}\right)$ and $(F,\|\cdot\|)$ are two Banach spaces with non empty intersection, then on the intersection space, $\mathcal{H}=E \cap F$, one can define the norm $\|\cdot\|_{\mathcal{H}}=\|\cdot\|_{E}+\|\cdot\|_{F}$. For our specific problem, given $p(\cdot), q(\cdot) \in C(\bar{\Omega})$ satisfying $(2.1)$, we introduce the function space $\mathcal{H}^{p(\cdot)}$ by:

$$
\mathcal{H}^{p(\cdot)}(\Omega)=X \cap W^{1, q(\cdot)}(\Omega),
$$

which can be equipped with the norm $\|\cdot\|=\|\cdot\|_{X}+\|\cdot\|_{1, q(\cdot)}$.

In this section, we establish the well-posedness of the proposed minimization problem.

Proposition 3.1. The following minimization problem

$$
\min _{u \in \mathcal{H}^{p(\cdot)}(\Omega)} \mathcal{J}(u)
$$

admits a unique minimizer $u$ in $\mathcal{H}^{p(\cdot)}(\Omega)$. Moreover, we have

$$
0<\inf _{\Omega} f \leq u \leq \sup _{\Omega} f
$$

Proof. First, it easy to see that the energy $\mathcal{J}(\cdot)$ is strictly convex and weakly lower semicontinuous in the space $\mathcal{H}^{p(\cdot)}(\Omega)$. Let us consider a minimizing sequence $\left(u_{n}\right)_{n} \subset H(\Omega):=$ $\left\{u>0, u \in \mathcal{H}^{p(\cdot)}(\Omega)\right\}$ of $\mathcal{J}(\cdot)$, i.e.,

$$
\mathcal{J}\left(u_{n}\right) \underset{n \rightarrow \infty}{\longrightarrow} \inf _{u \in \mathcal{H}^{p(\cdot)}(\Omega)} \mathcal{J}(u) .
$$

We denote by $\underline{m}:=\inf _{\Omega} f$ and $\bar{M}:=\sup _{\Omega} f$ and let $\left(v_{n}\right)_{n} \subset H(\Omega)$ be the sequence defined by $v_{n}=\min \left(u_{n}, \bar{M}\right)$ and set $D=\left\{x \in \Omega ; u_{n}(x) \geq \bar{M}\right\}$. Since on $\Omega \backslash D$ we have $u_{n}=v_{n}$, it follows that

$$
\begin{gathered}
\mathcal{J}\left(u_{n}\right)-\mathcal{J}\left(v_{n}\right)=\alpha \int_{D} \frac{1}{p(x)}\left|\Delta v_{n}\right|^{p(x)} \mathrm{d} x+\beta \int_{D} \frac{1}{q(x)}\left|\nabla u_{n}\right|^{q(x)} \mathrm{d} x \\
+\lambda \int_{D}\left(W\left(u_{n}\right)-W(\bar{M})\right) \mathrm{d} x .
\end{gathered}
$$


Using the convexity property of $W$ and the second inequality in (3.2), we have:

$$
\int_{D}\left(W\left(u_{n}\right)-W(\bar{M})\right) \mathrm{d} x \geq \int_{D} D_{u} W(M)\left(u_{n}-\bar{M}\right) \mathrm{d} x \geq 0 .
$$

It follows that $\mathcal{J}\left(v_{n}\right) \leq \mathcal{J}\left(u_{n}\right)$ and $\left(v_{n}\right)_{n}$ is also a minimizing sequence satisfying $v_{n} \leq M$. The same analysis goes for $w_{n}=\max \left(u_{n}, \underline{m}\right)$ and we also obtain that $\left(w_{n}\right)_{n}$ satisfying $\underline{m} \leq w_{n}$. Therefore, we can assume, without restriction, that

$$
u_{n} \in \mathcal{S}:=\left\{u \in \mathcal{H}^{p(\cdot)}(\Omega) ; \underline{m} \leq u(\cdot) \leq \bar{M}, \text { a.e. in } \Omega\right\} .
$$

Now, consider a minimizing sequence $\left(u_{n}\right)_{n} \in \mathcal{S}$. Then, we can easily see that $\left\|u_{n}\right\|_{L^{2}(\Omega)}$ is uniformly bounded. Using Proposition 2.1-a) and the fact $1<p(\cdot), q(\cdot) \leq 2$, we get that $\left\|u_{n}\right\|_{L^{p(\cdot)}}$ and $\left\|u_{n}\right\|_{L^{q(\cdot)}}$ are uniformly bounded. In addition, we have that $J^{p, q}\left(u_{n}\right) \leq C$, which implies, using Proposition 2.1-b) for $A\left(u_{n}\right)=\nabla u_{n}$ and $A\left(u_{n}\right)=\Delta u_{n}$, that $\left\|\Delta u_{n}\right\|_{L^{p(\cdot)}}$ and $\left\|\nabla u_{n}\right\|_{L^{g(\cdot)}}$ are uniformly bounded. Thus, we obtain that $\left(u_{n}\right)_{n}$ is uniformly bounded in $\mathcal{H}^{p(\cdot)}(\Omega)$ which means that there exists a subsequence, still denoted $\left(u_{n}\right)_{n \in \mathbb{N}}$, such that $u_{n} \underset{n \rightarrow \infty}{\rightarrow} u$ weakly in $\mathcal{H}^{p(\cdot)}(\Omega)$ and that the limit $u$ is a minimizer of $\mathcal{J}(\cdot)$. As the subset $\mathcal{S}$ is weakly closed, we have the limit $u \in \mathcal{S}$. The uniqueness comes from the strict convexity of $\mathcal{J}(\cdot)$.

The solution $u$ of the minimization problem (3.3) is a weak solution of the EulerLagrange equation:

$$
\begin{cases}\alpha \Delta\left(|\Delta u|^{p(x)-2} \Delta u\right)-\beta \operatorname{div}\left(|\nabla u|^{q(x)-2} \nabla u\right)+\lambda D_{u} W(u, f)=0, & \text { in } \Omega, \\ \frac{\partial}{\partial n}\left(|\Delta u|^{p(x)-2} \Delta u\right)=\frac{\partial u}{\partial n}=0, & \text { on } \partial \Omega .\end{cases}
$$

The operator $\Delta_{p(\cdot)}^{2} u:=\Delta\left(|\Delta u|^{p(x)-2} \Delta u\right)$ is of fourth-order and usually called the $p(\cdot)$ biharmonic operator $[1,26]$. In the particular case where $p(\cdot) \equiv q(\cdot) \equiv 2$, we get a linear fourth-order PDEs corresponding to the bi-Laplace operator. As mentioned in the introduction, we will consider the topological gradient for the particular case $p(\cdot) \equiv q(\cdot) \equiv 2$.

\section{Detection of important features via the topological gradient method}

A thin structure, like a filament in 3-D or a point in 2-D, is an object that can be linked to a curve. The usual spatial gradient is classically used for the edge detection. However, it is not efficient for thin structures [56,57]. Thin structures have a profile in the form of the delta function, whereas edges have a profile in the form of the Heaviside function. The edges have a jump of intensity through the discontinuity but the thin structure does not have a jump of intensity through the discontinuity more precisely the size of thin structures no greater than few pixels. The difficulties comes from the presence of noise and 
the fact that filaments are thin or/and non regular. Fortunately, the topological gradient approach provides an accurate identification of these kind of discontinuities $[6,22]$.

In the sequel, we recall the basic idea of the topological gradient method. For a small parameter $\varepsilon>0$, let $\Omega_{\varepsilon}$ be the perturbed domain of $\Omega$ obtained by creating a small hole $\omega_{\varepsilon}=\varepsilon \omega$ around the point $x \in \Omega$, i.e., $\Omega_{\varepsilon}=\Omega \backslash \omega_{\varepsilon}$, where $\omega$ is the fixed smooth open bounded subset in $\mathbb{R}^{2}$. Let $J_{\varepsilon}\left(u_{\varepsilon}\right)$ be a cost functional where $u_{\varepsilon}$ is a solution of a given PDE on the perturbed domain $\Omega_{\varepsilon}$. Note that $J_{0}(u)$ where $u$ is the solution of the given PDE on the initial domain $\Omega$. The variation of the cost functional, has the following asymptotic expansion [55]:

$$
J_{\varepsilon}\left(u_{\varepsilon}\right)-J_{0}(u)=\rho(\varepsilon) G\left(x_{0}\right)+o(\rho(\varepsilon)) .
$$

In this expansion, $\rho$ is an explicit function such that $\rho(\varepsilon) \geq 0$ and $\lim _{\varepsilon \rightarrow 0} \rho(\varepsilon)=0, G\left(x_{0}\right)$ is the topological gradient which does not depend on $\varepsilon$. To minimize the criterion $J_{\varepsilon}(\cdot)$, one has to create holes at some points where the topological gradient $G$ is negative, which correspond to regions containing important features to be detected.

In our approach, we define the following Fréchet differentiable cost functional which will be minimized outside the important features:

$$
J(u)=\frac{\alpha}{2} \int_{\Omega}|\Delta u|^{2} \mathrm{~d} x+\frac{\beta}{2} \int_{\Omega}|\nabla u|^{2} \mathrm{~d} x,
$$

where $\alpha$ and $\beta$ are positive parameters. The choice of this cost functional has two motivations. First, second-order derivatives are mostly used in order to detect and preserve thin structures, points or filaments $[5,6,22]$, where there is no jump across the intensity. Second, the classical gradient (first-order derivatives) usually gives a promising result in edge detection $[3,4,7,39]$. The parameters $\alpha$ and $\beta$ can be chosen so that either an edge or thin-structure is privileged.

We assume that important features are modeled by cracks and that the perturbed domain $\left(\Omega_{\varepsilon}\right)_{\varepsilon \geq 0}$ is obtained by inserting a small family of insulating cracks $\left(\sigma_{\varepsilon}\right)_{\varepsilon \geq 0}$, where $\sigma_{\varepsilon}=x_{0}+\varepsilon \sigma(n)$ and $\sigma$ is a straight segment centered at the origin and with normal $n$ (is the fixed crack) in $\Omega$. We denote by $\tau$ the tangent vector to the crack $\sigma$ such that $(n, \tau)$ forms a direct orthonormal basis and by $\mathrm{d} \tau$ the curvilinear Lebesgue measure along $\sigma$.

The solution $u_{\varepsilon}$ of the previous minimization problem fulfills the following optimality conditions:

$$
\begin{cases}\alpha \Delta^{2} u_{\varepsilon}-\beta \Delta u_{\varepsilon}+\lambda D_{u} W\left(u_{\varepsilon}, f\right)=0, & \text { in } \Omega_{\varepsilon}, \\ \frac{\partial \Delta u_{\varepsilon}}{\partial n}=\frac{\partial u_{\varepsilon}}{\partial n}=0, & \text { on } \partial \Omega, \\ \frac{\partial \Delta u_{\varepsilon}}{\partial n}=\Delta u_{\varepsilon}=0, & \text { on } \sigma_{\varepsilon},\end{cases}
$$

where $\Delta^{2}:=\Delta . \Delta$ is the bi-Laplace operator and $\partial \Omega_{\varepsilon}=\partial \Omega \cup \bar{\sigma}_{\varepsilon}$.

The computation of the topological gradient for the cost function (4.1) is a straightforward application of the analysis given in $[5,6,22,23,38]$. When calculating the topological gradient for (4.2), the second-order PDE will be dominated by the bi-Laplace operator and in this case we can not compute the topological gradient for the second-order PDEs. 
For this reason, we give in the following proposition the topological gradient for the second- and fourth-order PDE separately.

Proposition 4.1. Let $u$ be the solution of (3.6) with $p(x) \equiv q(x) \equiv 2$. Then, the topological gradient associated with the cost function (4.1) is given by:

- If $\beta=0$, we have the following topological gradient for a fourth-order PDE:

$$
G^{F}\left(x_{0}, n\right)=-\alpha \frac{2 \pi}{3} \nabla^{2} u\left(x_{0}\right)(n, n) \nabla^{2} \phi\left(x_{0}\right)(n, n),
$$

where $\phi$ solves the following adjoint equation:

$$
\begin{cases}\alpha \Delta^{2} \phi+\lambda D_{u}^{2} W(u, f) \phi=-\alpha \Delta^{2} u, & \text { in } \Omega, \\ \frac{\partial \Delta \phi}{\partial n}=\frac{\partial \phi}{\partial n}=0, & \text { on } \partial \Omega .\end{cases}
$$

- If $\alpha=0$, we have the following topological gradient for a second-order PDE (with the Neumann boundary condition on $\partial \Omega_{\varepsilon}$ ):

$$
G^{S}\left(x_{0}, n\right)=-\beta \pi \nabla u\left(x_{0}\right) \cdot n \nabla \phi\left(x_{0}\right) \cdot n,
$$

where $\phi$ solves the following adjoint equation:

$$
\begin{cases}-\beta \Delta \phi+\lambda D_{u}^{2} W(u, f) \phi=\beta \Delta u, & \text { in } \Omega, \\ \frac{\partial \phi}{\partial n}=0, & \text { on } \partial \Omega,\end{cases}
$$

with $D_{u}^{2} W(s, z)$ is the second derivative of $s \mapsto W(s, z)$ evaluated at $s \in \mathbb{R}_{+}^{*}$.

Remark 4.1. The adjoint equation (4.4) comes from the first-order optimality condition for the Lagrangian:

$$
\mathcal{L}(u, \phi)=J(u)+\alpha \int_{\Omega} \Delta u \Delta \phi \mathrm{d} x+\beta \int_{\Omega} \nabla u \nabla \phi \mathrm{d} x+\lambda \int_{\Omega} D_{u} W(u, f) \phi \mathrm{d} x .
$$

Equation (4.4) can be directly obtained by taking $\frac{\partial \mathcal{L}}{\partial u}(u, \phi)=0$ and applying Green's formula. For background materials we refer the reader to, e.g. [16,30,46,55]. Moreover, the adjoint equation is linear and by applying the Lax-Milgram theorem, it admits a unique solution $\phi \in H^{2}(\Omega)$ if $\beta=0$ and $\phi \in H^{1}(\Omega)$ if $\alpha=0$.

The quantity $G^{S}\left(x_{0}, n\right)$ corresponds to the topological gradient result of first-order derivatives (i.e., $\alpha=0$ ) and it is sensitive to edges (see $[7,39])$. It can be written as:

$$
G^{S}\left(x_{0}, n\right)=\langle M(x) n, n\rangle_{E}
$$

where $\langle\cdot, \cdot\rangle_{E}$ is the Euclidean inner product, $n=(\cos \theta, \sin \theta)$ is a unit normal to the crack with $\theta \in[0, \pi]$ and $M(x)$ is the $2 \times 2$ symmetric matrix defined by:

$$
M(x)=-\pi \beta \frac{\nabla u(x) \nabla \phi(x)^{T}+\nabla \phi(x) \nabla u(x)^{T}}{2} .
$$


On each point $x_{0}, G^{S}\left(x_{0}, n\right)$ takes its minimal value when $n$ is the eigenvector associated to the smallest eigenvalue of $M$. This value will be considered as the topological gradient indicator associated with the optimal orientation of the crack which corresponds to an edge of the image.

However, the quantity $G^{F}\left(x_{0}, n\right)$ is the topological gradient for fourth-order derivatives (i.e., $\beta=0$ ) and it is sensitive to thin structures and points. It has been proven in [22] that the quantity $G^{F}\left(x_{0}, n\right)$ can be written as:

$$
G^{F}\left(x_{0}, \theta\right)=-\alpha \frac{2 \pi}{3} P\left(\partial_{x x} u\left(x_{0}\right), \partial_{y y} u\left(x_{0}\right), \partial_{x y} u\left(x_{0}\right), \theta\right) P\left(\partial_{x x} \phi\left(x_{0}\right), \partial_{y y} \phi\left(x_{0}\right), \partial_{x y} \phi\left(x_{0}\right), \theta\right),
$$

where $P: \mathbb{R}^{4} \rightarrow \mathbb{R}$ is defined by:

$$
P(x, y, z, \theta)=\frac{1}{2}(x+y)+\frac{1}{2}(x-y) \cos (2 \theta)+z \sin (2 \theta),
$$

which is clearly a $\pi$-period function with respect to $\theta$.

Remark 4.2. The topological gradient method was studied in $[2,12,23,37,38]$ for a semilinear Laplace equations (more precisely, equation (4.2) for $\alpha=0$ ), and in [6] for the biLaplace operator with a linear second-member. The analysis in our case requires some tedious modifications of the result in [6]. For the reader's convenience and for the shake of completeness, we give the details in Appendix A.

In order to deal with the inequality constraint (3.4), we consider the following minimization problem:

$$
\min _{u} J^{p, q}(u)+\lambda \int_{\Omega} \frac{(f-u)^{2}}{u} \mathrm{~d} x+\iota_{C}(u),
$$

where $\iota_{C}$ is the indicator function of $C=[\underline{m}, \bar{M}]$ and which is given by:

$$
\iota_{C}(u)= \begin{cases}0, & \text { if } u \in C, \\ +\infty, & \text { if } u \notin C .\end{cases}
$$

The proof of existence and uniqueness of a minimizer for the energy (4.6) can be found in Appendix B.

\section{Implementation}

The smoothness of the solution is driven by the variable exponents $p(\cdot)$ and $q(\cdot)$ which will be chosen adaptively in order to slow diffusion near edges in the aim to sharpening and highlighting them, and to enhance diffusion in homogeneous regions. The restoration task is carried out in two steps: In an initial step, we use the topological gradient method for the biharmonic cost function (i.e., for $p(\cdot) \equiv q(\cdot) \equiv 2$ ), to detect important features. In a second step, we use the information furnished by the topological gradient 
(calculated for $p(\cdot) \equiv q(\cdot) \equiv 2$ ) in order to vary the exponent $p(\cdot)$ in the restoration process by considering a $p(\cdot)$-biharmonic model for $1<p(\cdot), q(\cdot) \leq 2$. The particularity of topological gradient of being an efficient edge- and thin structure-detector makes it well suited to control and locally select the exponent using the following algorithm:

Data: Given $f$ and $\lambda$.

1. For $p(\cdot) \equiv 2$ and $q(\cdot) \equiv 2$, compute $u$ and $v$ which solve equations (4.2) and (4.4), respectively.

2. Compute the topological gradient $G\left(x_{0}, n\right)$ for each point $x_{0} \in \Omega$.

3. Update $p(\cdot)$ and $q(\cdot)$ to obtain new exponents $p_{a}(\cdot)$ and $q_{a}(\cdot)$. Then, solve (3.6) for $p(\cdot)=p_{a}(\cdot)$ and $q(\cdot)=q_{a}(\cdot)$.

Algorithm 1: Main algorithm

In order to update the exponents $p(\cdot)$ and $q(\cdot)$, we use the following formulas:

$$
p_{a}(x)=1+\exp \left(-\kappa_{1}\left|G^{F}(x, n)\right|\right), q_{a}(x)=1+\exp \left(-\kappa_{2}\left|G^{S}(x, n)\right|\right), \quad \forall x \in \Omega,
$$

where $\kappa_{1}, \kappa_{2}$ are positive constants. In homogeneous regions, we have $G^{F}(x, n) \approx 0$, (respectively $G^{S}(x, n) \approx 0$ ) leading to a new exponent $p_{a}(x)$ (respectively $q_{a}(x)$ ) close to 2 . Then, the model behaves like the biharmonic equation leading to strong diffusion and hence noise is damped. Near edges, $G^{F}(x, n)$ (respectively $G^{S}(x, n)$ ) is very important and therefore $p_{a}(x)$ (respectively $q_{a}(x)$ ) will be close to 1 which slows down diffusion. However, for such a choice of $p_{a}(\cdot)$ and $q_{a}(\cdot)$, equation (3.6) is strongly nonlinear. For that, we will use a split Bregman algorithm to numerically solve it.

\subsection{Split-Bregman Algorithm}

In [35], the authors proposed a new technique based on the Bregman iteration for solving non-smooth problems, particularly, $L^{1}$ - regularized problems. Originally, it was invented to solve the Rudin, Osher and Fatemi (ROF) model in image restoration. See also the works by Getreuer [31-33] which use the split-Bregman method for TV denoising, deblurring and inpainting. Then, it was applied for more general problems such as higherorder models. In our case, the original energy functional (1.6) is a non-differentiable functional of $u$.

We assume that our discrete images have $r \times c$ pixels, where $r$ and $c$ are the numbers of rows and columns in the image, respectively. We define the discrete operators and norms that will be used in the numerical implementation. We first consider the following 
discrete norms:

$$
\begin{cases}\|u\|_{2}=\left(\sum_{i=1}^{r} \sum_{j=1}^{c} u(i, j)^{2}\right)^{1 / 2}, & u:\{1, \cdots, r\} \times\{1, \cdots, c\} \longrightarrow \mathbb{R}, \\ \|m\|_{p}=\sum_{i=1}^{r} \sum_{j=1}^{c} \frac{1}{p(i, j)}|m(i, j)|^{p(i, j)}, & m:\{1, \cdots, r\} \times\{1, \cdots, c\} \longrightarrow \mathbb{R}, \\ \|\mathbf{n}\|_{q}=\sum_{i=1}^{r} \sum_{j=1}^{c} \frac{1}{q(i, j)}\left(n_{1}(i, j)^{2}+n_{2}(i, j)^{2}\right)^{q(i, j) / 2}, & \mathbf{n}:\{1, \cdots, r\} \times\{1, \cdots, c\} \longrightarrow \mathbb{R}^{2} .\end{cases}
$$

For the discrete differential operators, we assume periodic boundary conditions for $u$. By choosing periodic boundary conditions, the action of each of the discrete differential operators can be regarded as a circular convolution of $u$ and allows the use of fast Fourier transform (see $[49,59,60]$ for more details). We consider the discrete first- and secondorder derivatives $D_{x}, D_{y}, D_{x x}$ and $D_{y y}$ as operators from $\mathbb{R}^{r \times c}$ to $\mathbb{R}$. The discrete gradient is $D u=\left(D_{x} u, D_{y} u\right)$ where $D_{x}$ and $D_{y}$ are forward difference operators.

In the sequel, we will use the split Bregman algorithm for solving the optimization problem (4.6). The main idea is to introduce auxiliary variables into the unconstrained optimization problem and then solve a constrained one which is more easy to handle. First, we rewrite the original optimization problem (4.6) in the following form:

$$
\min _{u \in \mathbb{R}^{\ell}} F(u)+\sum_{k=1}^{3} G_{k}\left(A_{k} u\right),
$$

where

$$
\left\{\begin{array}{l}
{\left[A_{1}, A_{2}, A_{3}\right]^{\mathrm{T}}=[\mathbf{I}, \Delta \cdot, \nabla \cdot]^{\mathrm{T}}, F(u)=\iota_{C}(u), \quad \forall u \in \mathbb{R}^{\ell}, \ell=r \times c,} \\
G_{1}(v)=\lambda \sum_{i=1}^{r} \sum_{j=1}^{c} \frac{(v(i, j)-f(i, j))^{2}}{v(i, j)}, \forall v \in \mathbb{R}^{\ell}, G_{2}(m)=\alpha\|m\|_{p}, \quad \forall m \in \mathbb{R}^{\ell}, \\
G_{3}(\mathbf{n})=\beta\|\mathbf{n}\|_{q}, \quad \forall \mathbf{n} \in \mathbb{R}^{2 \ell} .
\end{array}\right.
$$

Then, the split-Bregman method applied to the problem (4.6) consists in solving the following constrained optimization problem:

$$
\min _{u \in \mathbb{R}^{\ell}} F(u)+G(X), \text { s.t., } \mathbf{A} u=X,
$$

where $\mathbf{A}=[\mathbf{I}, \Delta \cdot, \nabla \cdot]^{\mathrm{T}}, X=[v, m, \mathbf{n}]^{\mathrm{T}}$ and $G(X)=G_{1}(v)+G_{2}(m)+G_{3}(\mathbf{n})$. The strong point of the split Bregman method is that it is easier to solve the constrained optimization problem (5.3) rather than the unconstrained optimization problem (5.2).

Remark 5.1. It appears on first sight that it is not necessary to introduce the auxiliary variable $v$. However, this auxiliary variable is crucial since it allows to avoid solving a nonlinear problem which will be faced in the $u$-subproblem due to the nonlinear data fitting term $W(u, f)$. 
The split Bregman algorithm applied to the constrained optimization problem (5.3) consists on the following iterations:

1 Initialization: $u_{1}=u_{0}, X_{1}=\left(v_{1}, m_{1}, \mathbf{n}_{1}\right)=\left(u_{0}, \Delta u_{0}, \nabla u_{0}\right), b_{0}^{0}=1, b_{0}^{1}=1$ and $b_{0}^{2}=1$.

2 Find $u_{k+1}$ and $X_{k+1}=\left(v_{k+1}, m_{k+1}, \mathbf{n}_{k+1}\right)$ which solve

$$
\begin{gathered}
{\left[u_{k+1}, X_{k+1}\right]=\underset{u, X}{\operatorname{argmin}} F(u)+G(X)+\frac{\lambda_{0}}{2}\left\|b_{k}^{0}+u-v\right\|_{2}^{2}+\frac{\lambda_{1}}{2}\left\|b_{k}^{1}+\Delta u-m\right\|_{2}^{2}} \\
+\frac{\lambda_{2}}{2}\left\|b_{k}^{2}+\nabla u-\mathbf{n}\right\|_{2}^{2}, X=(v, m, \mathbf{n}),
\end{gathered}
$$

3 Update

$$
\begin{aligned}
& b_{k+1}^{0}=b_{k}^{0}+u_{k+1}-v_{k+1}, \\
& b_{k+1}^{1}=b_{k}^{1}+\Delta v_{k+1}-m_{k+1}, \\
& b_{k+1}^{2}=b_{k}^{2}+\nabla v_{k+1}-\mathbf{n}_{k+1} .
\end{aligned}
$$

\section{Algorithm 2: Split-Bregman iterations}

Remark 5.2. The proposed problem is splited into two blocks. The convergence proof of the two-block split Bregman method can be found in $[15,35,48,52,54]$. In our case, as $F(\cdot)$ and $G_{k}(\cdot),(k=1,2,3)$ in the optimization problem (5.2) are proper, convex and lower semi-continuous functionals, the convergence can easily be proved following the same line of proof in [54]. Thus, the sequence $\left(u_{k}\right)$ obtained by the $u$-subproblem converges to the solution of the original minimization problem (4.6).

It is difficult to minimize the energy (5.4) with respect to all variables jointly. Thus in every iteration, we split it into four separate subproblems, each of which can be quickly solved. We apply an alternating minimization iterative procedure, namely, for $k=0,1, \ldots$, we solve sequentially:

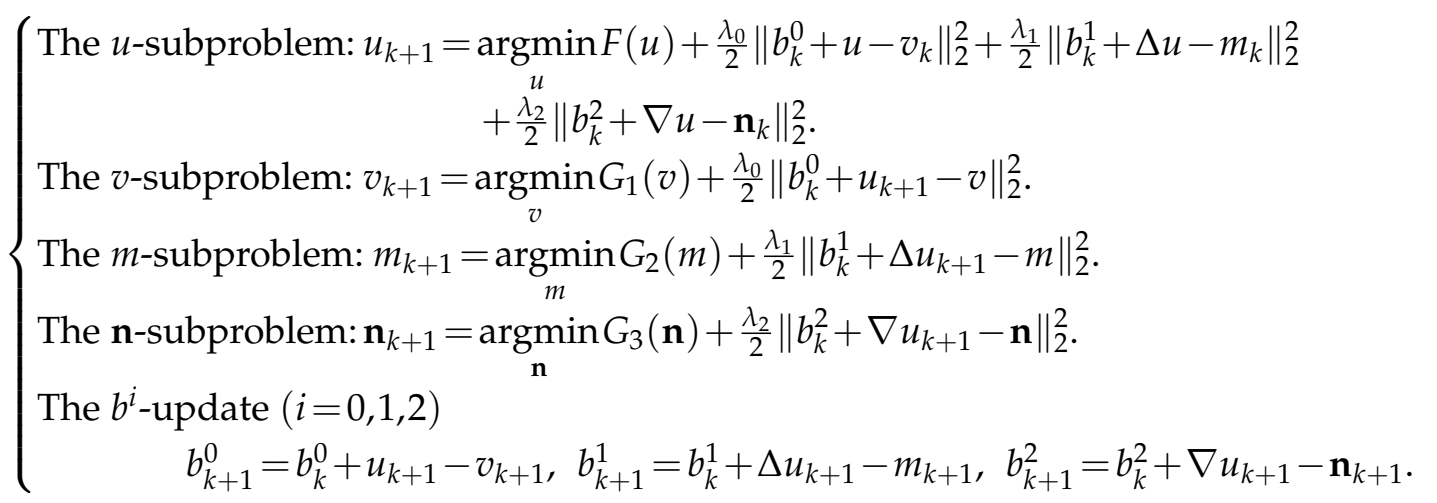


The $u$-subproblem: This problem is

$$
u_{k+1}=\underset{u \in \mathbb{R}^{\ell}}{\operatorname{argmin}} F(u)+\frac{\lambda_{0}}{2}\left\|b_{k}^{0}+u-v_{k}\right\|_{2}^{2}+\frac{\lambda_{1}}{2}\left\|b_{k}^{1}+\Delta u-m\right\|_{2}^{2}+\frac{\lambda_{2}}{2}\left\|b_{k}^{2}+\nabla u-\mathbf{n}\right\|_{2}^{2} .
$$

Remark 5.3. Solving the above $u$-subproblem is a non-trivial task because of the the presence of the term $F(u)$. Such a problem can be handled by using the Moreau-Yosida penalty function method. However, this $u$-subproblem will lead to a semi-linear PDE which can be solved using different algorithms such as fixed-point iterations, Newton method [58] and time-marching method that are known to be very slow. Here, we choose to use an inner split Bregman iterations which allows us to obtain two easy sub-problems. In fact, the first problem can be solved easily using the FFT transform, and the solution of the second problem is explicitly given by a proximity operator [14].

Here, we will use an inner split-Bregman method to solve the $u$-subproblem by introducing a variable $w$ and we solve

$$
\begin{aligned}
u_{k+1}=\underset{u \in \mathbb{R}^{\ell}}{\operatorname{argmin}} & F(w)+\frac{\lambda_{0}}{2}\left\|b_{k}^{0}+u-v_{k}\right\|_{2}^{2}+\frac{\lambda_{1}}{2} \| b_{k}^{1} \\
& \quad+\Delta u-m\left\|_{2}^{2}+\frac{\lambda_{2}}{2}\right\| b_{k}^{2}+\nabla u-\mathbf{n} \|_{2}^{2}, \text { such that, } w=u .
\end{aligned}
$$

We introduced the auxiliary variable $w$ in order to avoid solving non classical PDE which will be faced in the $u$-subproblem. For this case, we can also use the following iterative algorithm to solve for the variables $v$ and $w$ in the above constrained $u$-subproblem

1 Initialization: $w_{0}=v_{1}$ and $b_{0}^{3}=0$.

2 Solve

$$
\begin{aligned}
u_{k+1}=\underset{u \in \mathbb{R}^{\ell}}{\operatorname{argmin}} \frac{\lambda_{0}}{2}\left\|b_{k}^{0}+u-v_{k}\right\|_{2}^{2}+\frac{\lambda_{1}}{2}\left\|b_{k}^{1}+\Delta u-m\right\|_{2}^{2}+\frac{\lambda_{2}}{2}\left\|b_{k}^{2}+\nabla u-\mathbf{n}\right\|_{2}^{2} \\
\quad+\frac{\lambda_{3}}{2}\left\|b_{k}^{3}+u_{k+1}-w\right\|_{2}^{2} . \\
w_{k+1}=\underset{w \in \mathbb{R}^{\ell}}{\operatorname{argmin}} F(w)+\frac{\lambda_{3}}{2}\left\|b_{k}^{3}+u^{k+1}-w\right\|_{2}^{2} .
\end{aligned}
$$

3 Update the Bregman iteration

$$
b_{k+1}^{3}=b_{k}^{3}+u_{k+1}-w_{k+1} .
$$

Algorithm 3: Split-Bregman iterations for the $u$-subproblem

The subproblems (5.5) and (5.6) can be efficiently solved. The problem (5.5) is quadratic and it is solved through its optimality condition given by the following fourth-order PDE:

$$
\lambda_{1} \Delta^{2} u-\lambda_{2} \Delta u+\left(\lambda_{0}+\lambda_{3}\right) u=\lambda_{0}\left(b_{k}^{0}-v_{k}\right)+\lambda_{3}\left(b_{k}^{3}-w_{k}\right)+\lambda_{1} \Delta\left(m_{k}-b_{k}^{1}\right)-\lambda_{2} \operatorname{div}\left(b_{k}^{2}-\mathbf{n}_{k}\right) .
$$


To solve the previous fourth-order PDE we use the 2-dimensional discrete Fourier transforms $\mathcal{F}$. In fact, we have:

$$
\mathcal{F}(u)(i, j)=\frac{\mathcal{F}\left(\lambda_{0}\left(b_{k}^{0}-v_{k}\right)+\lambda_{3}\left(b_{k}^{3}-w_{k}\right)-\lambda_{2} \operatorname{div}\left(b_{k}^{2}-\mathbf{n}_{k}\right)\right)+\lambda_{1} M_{i, j} m_{k}}{\lambda_{1} M_{i, j}^{2}-\lambda_{2} M_{i, j}+\left(\lambda_{0}+\lambda_{3}\right) \delta_{i j}},
$$

where $M_{i, j}$ is the discrete Fourier of the operator $\Delta$ and which is given by:

$$
M_{i, j}=\frac{2}{(\Delta x)^{2}}\left(\cos \left(\frac{2 \pi i}{r}\right)-1\right)+\frac{2}{(\Delta y)^{2}}\left(\cos \left(\frac{2 \pi j}{c}\right)-1\right),
$$

where $\Delta x$ and $\Delta y$ are the mesh size in the $x$ - and $y$-directions, respectively, see for instance $[34,49]$.

Therefore, the discrete solution $u$ can be obtained by applying the inverse of the discrete two-dimensional Fourier transform $\mathcal{F}^{-1}$ to equation (5.7).

The solution $w$ of (5.6) is given by the proximal operator

$$
w=\operatorname{prox}_{\frac{\left.i_{C}(w)\right)}{\lambda_{3}}}\left(b_{k}^{3}+u_{k+1}\right)= \begin{cases}b_{k}^{3}+u_{k+1}, & \text { if } b_{k}^{3}+u_{k+1} \in[\underline{m}, \bar{M}], \\ \bar{M}, & \text { if } b_{k}^{3}+u_{k+1}>\bar{M}, \\ \underline{m} & \text { if } b_{k}^{3}+u_{k+1}<\underline{m} .\end{cases}
$$

The $v$-subproblem: This problem is

$$
v_{k+1}=\underset{v \in \mathbb{R}^{\ell}}{\operatorname{argmin}} G_{1}(v)+\frac{\lambda_{0}}{2}\left\|b_{k}^{0}+u_{k+1}-v\right\|_{2}^{2}
$$

whose solution fulfills:

$$
A v(i, j)^{3}+B v(i, j)^{2}+C=0,
$$

where $A=\lambda_{0}, B=\lambda+\lambda_{0}\left(b_{k}^{0}(i, j)+u_{k+1}(i, j)\right)$ and $C=-\lambda f(i, j)^{2}$. The polynomial

$$
P(X)=A X^{3}+B X^{2}+C,
$$

is of degree 3 and hence has at least one real root. However, in our case, we can prove that it has exactly one real root which corresponds to the solution of (5.9). In fact, every root of $P(\cdot)$ is a solution of (5.9) which clearly is unique, because the strict convexity of the energy functional.

The $m$-subproblem: This problem is

$$
m_{k+1}=\underset{m \in \mathbb{R}^{\ell}}{\operatorname{argmin}} G_{2}(m)+\frac{\lambda_{1}}{2}\left\|b_{k}^{1}+\Delta u_{k+1}-m\right\|_{2}^{2},
$$

whose minimizer $m_{k+1}$ is given explicitly by the following shrinkage-like formula [8]:

$$
m_{k+1}=\max \left\{\left|b_{k}^{1}+\Delta u_{k+1}\right|-\frac{\alpha\left|b_{k}^{1}+\Delta u_{k+1}\right|^{p(\cdot)-1}}{\lambda_{1} p(\cdot)}, 0\right\} \frac{b_{k}^{1}+\Delta u_{k+1}}{\left|b_{k}^{1}+\Delta u_{k+1}\right|},
$$

where $\frac{0}{0}=0$ is assumed. 
The n-subproblem: This problem is

$$
\mathbf{n}_{k+1}=\underset{\mathbf{n} \in \mathbb{R}^{2 \ell}}{\operatorname{argmin}} G_{3}(\mathbf{n})+\frac{\lambda_{2}}{2}\left\|b_{k}^{2}+\nabla u_{k+1}-\mathbf{n}\right\|_{2}^{2}
$$

whose minimizer $\mathbf{n}_{k+1}$ is given explicitly by the following shrinkage-like formula:

$$
\mathbf{n}_{k+1}=\max \left\{\left\|b_{k}^{2}+\nabla u_{k+1}\right\|_{2}-\frac{\beta\left\|b_{k}^{2}+\nabla u_{k+1}\right\|_{2}^{q(\cdot)-1}}{\lambda_{2} q(\cdot)}, 0\right\} \frac{b_{k}^{2}+\nabla u_{k+1}}{\left\|b_{k}^{2}+\nabla u_{k+1}\right\|_{2}},
$$

where $\|\cdot\|_{2}$ is the Euclidean norm.

\section{Numerical experiments}

In the following section, we use some numerical experiments to examine the efficiency and robustness of the Algorithm 5.1. All numerical experiments were run for noisy gray images to recover the original ones. All the images used here were downloaded from the internet and are significantly related to the purpose of this work which is to detect edges and thin structures from medical images. The performance of the proposed approach is illustrated for multiplicative speckle noise removal and important features detection. We compare our model with the following classical ones:

- The TVL model i.e., $p(\cdot) \equiv q(\cdot) \equiv 1$ :

$$
\begin{gathered}
\min _{u, v, m, \mathbf{n}} \alpha\|m\|_{1}+\beta\|\mathbf{n}\|_{1}+\lambda \sum_{i=1}^{r} \sum_{j=1}^{c} \frac{(v(i, j)-f(i, j))^{2}}{v(i, j)}+\iota_{C}(u), \\
\text { such that } u=v, \Delta u=m, \nabla u=\mathbf{n} .
\end{gathered}
$$

- The biharmonic model i.e., $p(\cdot) \equiv q(\cdot) \equiv 2$ :

$$
\begin{gathered}
\min _{u, v, m, \mathbf{n}} \alpha\|m\|_{2}+\beta\|\mathbf{n}\|_{2}+\lambda \sum_{i=1}^{r} \sum_{j=1}^{c} \frac{(v(i, j)-f(i, j))^{2}}{v(i, j)}+\iota_{C}(u), \\
\text { such that } u=v, \Delta u=m, \nabla u=\mathbf{n} .
\end{gathered}
$$

We also used the split-Bregman algorithm to solve the two problems (6.1) and (6.2). For all models, we stop the split-Bregman iterations when the following stopping criterion $\frac{\left\|u^{k+1}-u^{k}\right\|}{\left\|u^{k}\right\|} \leq 10^{-3}$ is satisfied. Denoising performance is evaluated using the Structural Similarity Index (SSIM) which measures the perceptual difference between the restored and the original images. The SSIM takes values between 0 and 1 , where a value close to one means that the noise is low. We also use the the Signal-to-Noise Ratio (SNR) and Peak Signal-to-Noise Ratio (PSNR) indicators (the higher SNR/PSNR is the better restoration 
is). For all numerical examples to be discussed below, we display the original image, noisy one, the topological gradient indicator for the second- and fourth-order PDE, and the variable-exponent functions $p(\cdot)$ and $q(\cdot)$.

In order to reduce the number of parameters to tune, we set $\lambda_{0}=\lambda_{3}=1, \lambda_{1}=2$ and $\lambda_{2}=5$ in all numerical experiments. The choice of the parameters $\alpha, \beta$, and $\lambda$ will affect how much the image is regularized. These parameters provide a balance between denoising and preserving the important objects and edges.

In the first example presented in Figure 1, we test the efficiency of the proposed approach in detecting thin-structures such as blood vessels and veins. We also compare all the competitive models and the optimal choice of the regularization parameters for all models were chosen after making different tests by setting $\alpha=0.2, \beta=0.3$ and varying $\lambda \in\{1,1.5,2,2.5,3,3.5,4,4.5,5\}$. We have found that the optimal parameters are $\lambda=3, \lambda=3$ and $\lambda=5$ for the biharmonic, $p(\cdot)$-Kirchhoff and the TVL models, respectively.

Figures 1(c) and 1(d) show the topological gradient indicators for the second- and fourth-order operators, respectively. The topological gradient (4.3) coming from the fourthorder derivatives performs well in detecting thin-structures. Whereas, the gradient (4.5) which is coming from second-order derivatives is effective only in detecting normal edges, i.e., not the edges a thin-structures.

The images 1(e) and 1(f) show the variable-exponent functions $q(\cdot)$ and $p(\cdot)$, respectively. Their values are ranging between 1 and 2 . Near important features, they took values close to 1 which means slowing down diffusion in the model, whereas the values are close to 2 elsewhere. We note that the topological gradients and the masks created by the exponents give a good description of the image objects, i.e., edges and thin structures. Moreover, we measure the quality of restoration using the SSIM, PSNR, and SNR indicators which confirm that our model; e.g. image 1(g) in Figure 1, gives better restoration results compared to the other models.

Figure 2 shows the convergence of the split-Bregman scheme for the three models. We display the curves of SSIM indicators as functions of split-Bregman iterations. The SSIM indicator for the TVL model converges monotonically and slowly to 0.84 after 101 iterations. This is not surprising since the $\Delta\left(\frac{\Delta \cdot}{|\Delta \cdot|_{\delta}}\right)$ and $\operatorname{div}\left(\frac{\nabla \cdot}{|\nabla \cdot|_{\delta}}\right)$ have low impact on smoothness. For the biharmonic model, the SSIM values tends to 0.83 after only 39 iterations and slightly decreases after 39 iterations (see Figure 2). This is expected because of the high-smoothness properties of the bi-Laplace operator, $\Delta^{2}$. If more iterations were performed, the image will be over-smoothed. However, the SSIM values for the $p(\cdot)$ Kirchhoff model rapidly tends to 0.84 and less iteration (48 iterations) are needed compared to the other models. 
In order to show the robustness of the topological gradient approach in detecting thin-structures such as filaments and points, we consider in Figure 3 an image which contains point-like objects. The fourth-order topological gradient is more efficient than the second-order topological gradient in detecting thin structures. This result highlights the importance of the high-order terms in detecting and preserving thin structures.

In Figures 4 and 5, we compared the three competitive models in denoising ultrasound medical images. In both examples, our model is more effective than the TVL and the biharmonic models in noise reduction as well as thin-structure preservation. The denoised images using the different models look visually similar. The main dissimilarity is highlighted by zooming in the middle parts of the images in Figure 7 where it is clear that the proposed approach outperforms the TVL and the biharmonic models. We give in Figure 6 the curve of the SSIM values, for the images in Figures 4 and 5, as a function of the level of noise $\sigma^{2}$. For $\sigma^{2}<0.06$, the three models are broadly comparable. However, the advantages of the proposed model can be seen for $\sigma^{2}>0.06$. We quantitatively compare all results using the SSIM, PSNR and SNR indicators which are summarized in Table 1.

\section{Conclusion}

In this paper, we have presented a new approach to restore images corrupted with speckle multiplicative noise. The proposed approach combines the advantages of the topological gradient method for the detection of important features and the diffusion proprieties of a nonstandard $p(\cdot)$-Kirchhoff operator for the denoising. A split Bregman algorithm is used to solve the proposed model. Different numerical examples and comparison with different models were performed. The results showed a good quality in image denoising and thin-structure preservation.

\section{A Proof of Proposition 4.1}

The topological gradient for the second-order semilinear PDE (i.e., $\alpha=0$ ) is given in [23, $37,38]$. Here, we only give the main steps of the proof in the case of the fourth-order semilinear PDE (i.e., $\beta=0$ ). For more details, see [6].

Table 1: Comparison between the $p(\cdot)$-Kirchhoff, $T V L$ and biharmonic models.

\begin{tabular}{||l|llll|lllll|lllll||}
\hline & \multicolumn{4}{|c|}{$p(\cdot)$-Kirchhoff } & \multicolumn{4}{c|}{ biharmonic } & \multicolumn{5}{c|}{ TVL } \\
\cline { 2 - 12 } & SSIM & PSNR & SNR & Iter. & SSIM & PSNR & SNR & Iter. & SSIM & PSNR & SNR & Iter. \\
\hline Figure 1 & 0.847 & 29.3 & 26.52 & 48 & 0.842 & 28.58 & 25.97 & 39 & 0.837 & 27.79 & 25.19 & 101 \\
\hline Figure 3 & 0.7 & 25.74 & 24.03 & 41 & 0.66 & 23.44 & 21.83 & 57 & 0.67 & 24.41 & 22.8 & 32 \\
\hline Figure 4 & 0.96 & 32.28 & 21.08 & 76 & 0.95 & 30.68 & 20.33 & 52 & 0.962 & 32.2 & 20.85 & 69 \\
\hline Figure 5 & 0.868 & 30 & 21.62 & 44 & 0.861 & 29.36 & 20.98 & 51 & 0.867 & 29.91 & 21.53 & 46 \\
\hline
\end{tabular}




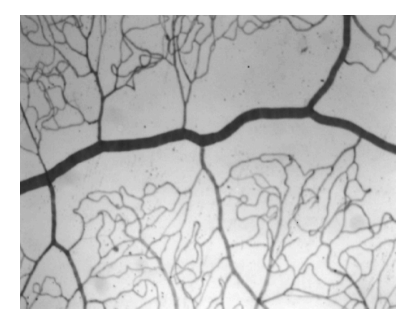

(a)

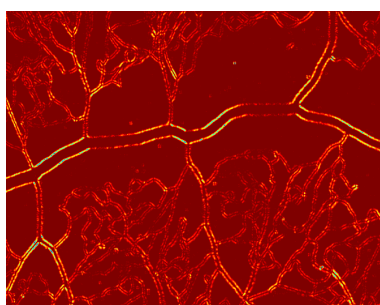

(c)

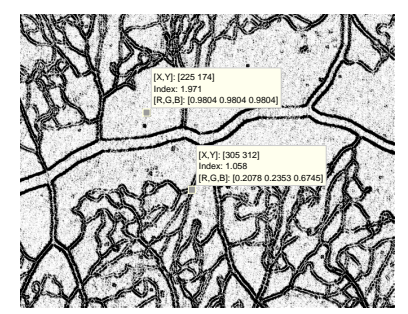

(e)

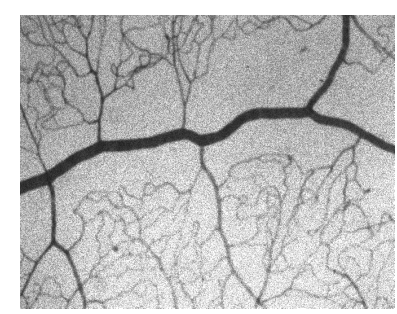

(b)

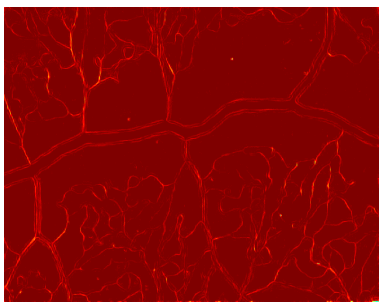

(d)

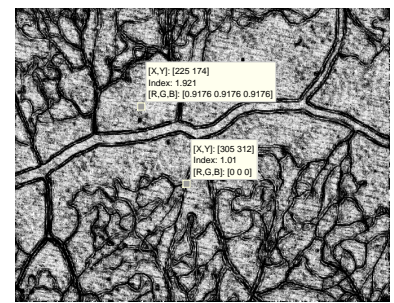

(f)

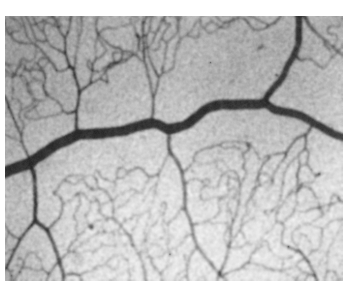

(g)

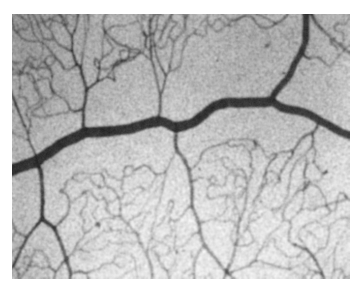

(h)

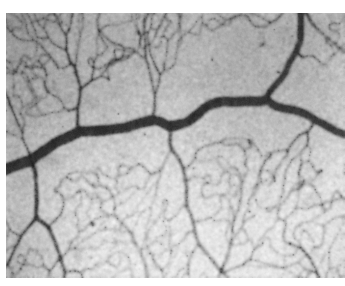

(i)

Figure 1: (a) Original image, (b) noisy one, (c) topological gradient for the second-order model, (d) topological gradient for the fourth-order model, (e) the variable exponent $q(\cdot)$, (f) the variable exponent $p(\cdot),(\mathrm{g}) p(\cdot)$ Kirchhoff model, (h) TVL model and (i) biharmonic model. 


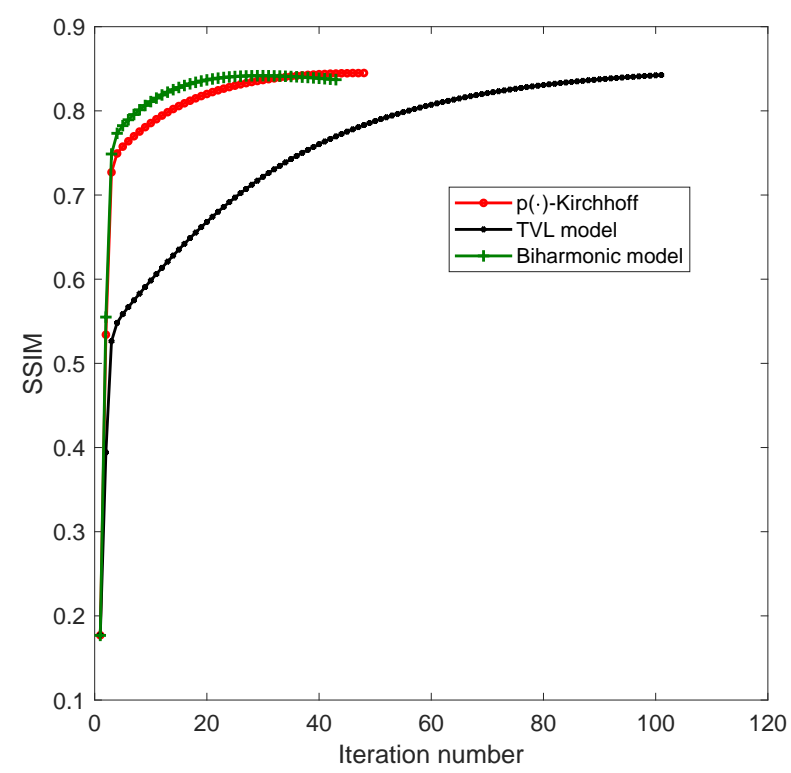

Figure 2: Convergence of the split-Bregman method (applied to the image in Figure 1).

\section{A.1 Exterior boundary-value problem}

Let $\Sigma$ a regular open manifold of dimension 1 included in $\mathbb{R}^{2}$. We denote by $W^{m}\left(\mathbb{R}^{2} \backslash \Sigma\right)$ the weighted Sobolev space defined by [47]:

$W^{m}\left(\mathbb{R}^{2} \backslash \bar{\Sigma}\right)=\left\{u, \frac{\nabla^{k} u}{\left(1+r^{2}\right)^{\frac{m-k}{2}} \log \left(2+r^{2}\right)} \in L^{2}\left(\mathbb{R}^{2} \backslash \bar{\Sigma}\right)\right.$ for $k \in\{0, \ldots, m-1\}$ and $\left.\nabla^{m} u \in L^{2}\left(\mathbb{R}^{2} \backslash \bar{\Sigma}\right)\right\}$,

where $r=|x|$. We denote by $W^{m}\left(\mathbb{R}^{2} \backslash \bar{\Sigma}\right) / \mathbb{P}_{m-1}$ is the quotient space of functions $W^{m}\left(\mathbb{R}^{2} \backslash\right.$ $\bar{\Sigma}$ ) defined up to a polynomial of degree less or equal than $m-1$.

Let us now introduce the following boundary value problem for the bi-Laplace problem defined in the weighted Sobolev space.

Theorem A.1. Given the auxiliary exterior-domain problem

$$
\begin{cases}\Delta^{2} H=0, & \text { on } \mathbb{R}^{2} \backslash \bar{\Sigma}, \\ \frac{\partial \Delta H}{\partial n}=g_{1}, & \text { in } \Sigma, \\ \Delta H=g_{2}, & \text { in } \Sigma,\end{cases}
$$

where $\left(g_{1}, g_{2}\right) \in\left(H_{00}^{\frac{3}{2}}(\Sigma)\right)^{\prime} \times\left(H_{00}^{\frac{1}{2}}(\Sigma)\right)^{\prime}$. We assume that $\Lambda=\mathbb{R}^{2} \backslash \bar{\Sigma}$ is connected. Then, equation (A.1) admits an unique solution $H \in W^{2}(\Lambda) / \mathbb{P}_{1}$ satisfying

$$
H(x)=\int_{\sigma} \Delta E(x-y) \eta(y) \mathrm{d} \sigma(y),
$$




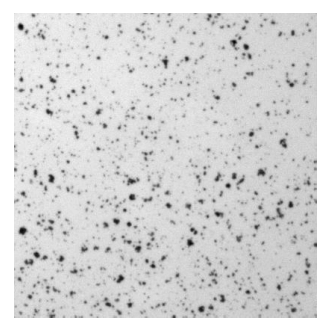

(a)

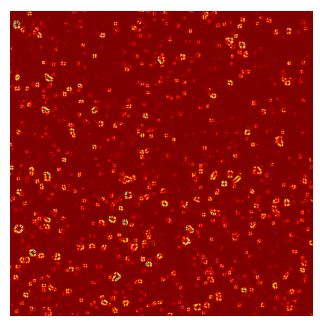

(c)

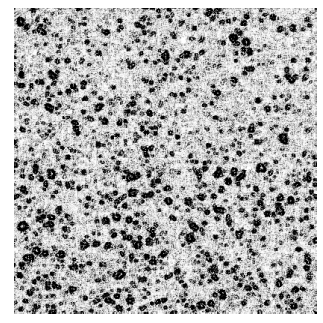

(e)

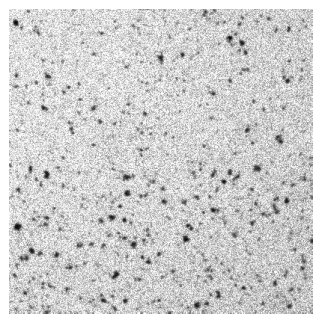

(b)

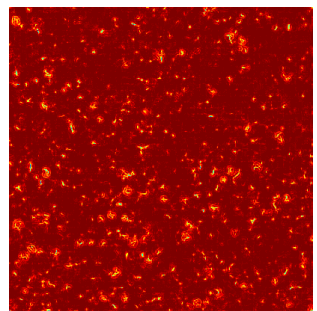

(d)

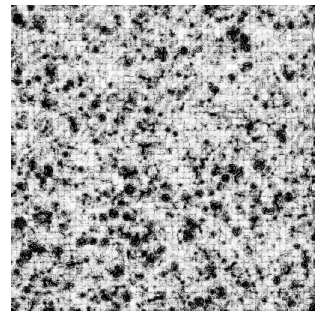

(f)

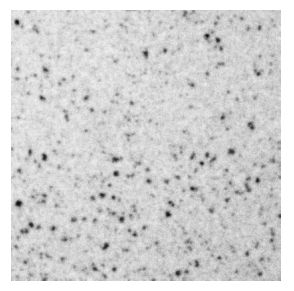

(g)

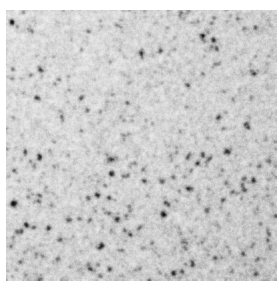

(h)

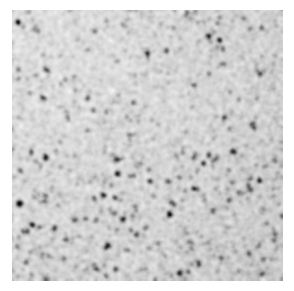

(i)

Figure 3: (a) Original image, (b) noisy one, (c) topological gradient for the second-order model, (d) topological gradient for the fourth-order, (e) the variable exponent $q(\cdot)$, (f) the variable exponent $p(\cdot),(\mathrm{g}) p(\cdot)$-Kirchhoff model, (g) TVL model and (i) biharmonic model, $(\alpha=0.2$ and $\beta=0.1)$. 


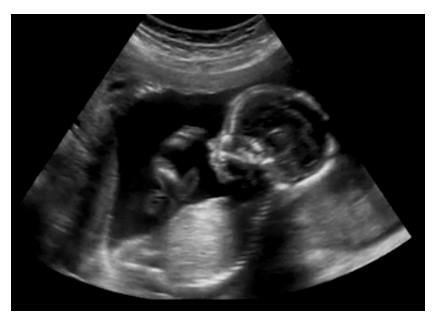

(a)

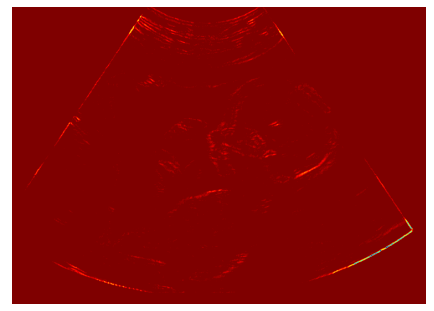

(c)

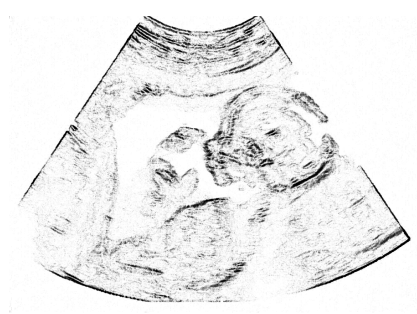

(e)

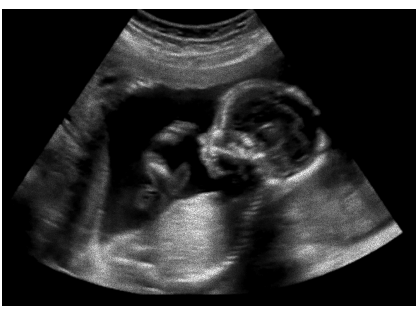

(b)

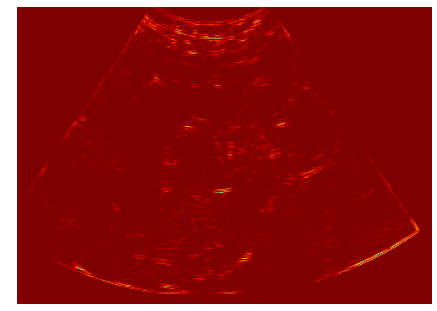

(d)

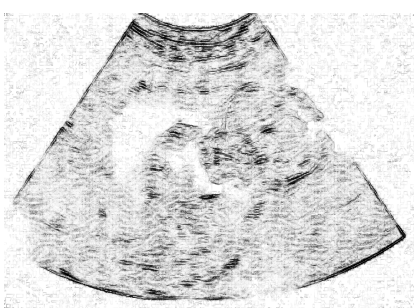

(f)

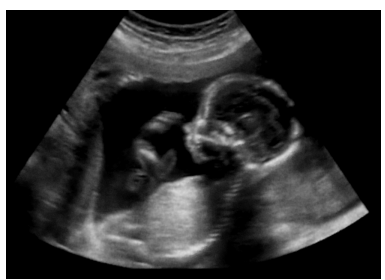

(g)

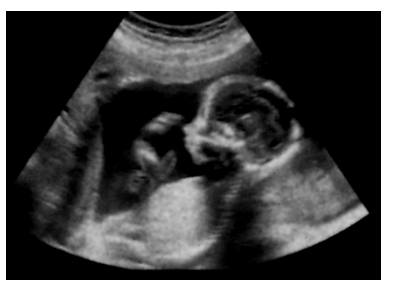

(h)

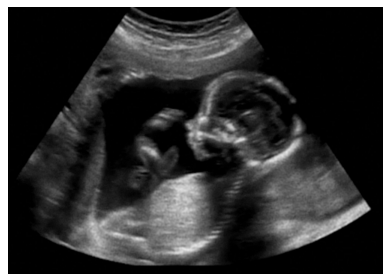

(i)

Figure 4: (a) Original image, (b) noisy one, (c) topological gradient for the second-order model, (d) topological gradient for the fourth-order model, (e) the variable exponent $q(\cdot)$, (f) the variable exponent $p(\cdot),(\mathrm{g}) p(\cdot)$ Kirchhoff model, (h) the TVL model and (e) the biharmonic model, $(\alpha=0.2$ and $\beta=0.2)$. 


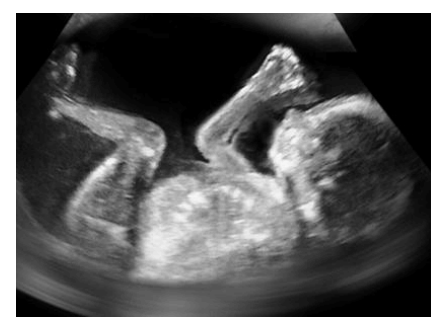

(a)

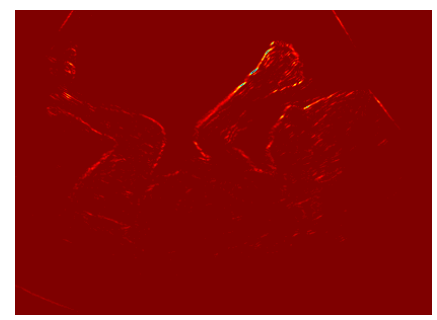

(c)

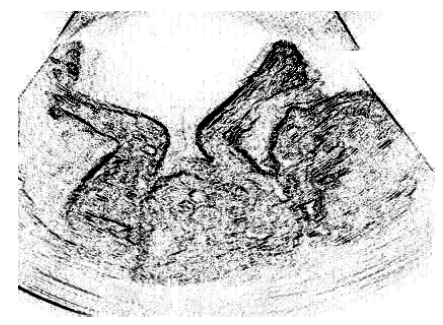

(e)

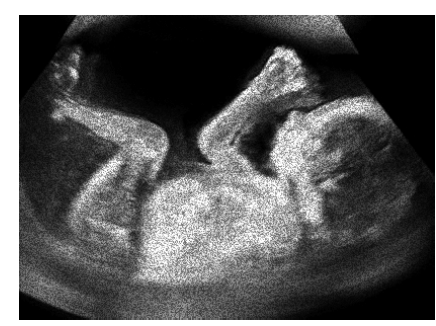

(b)

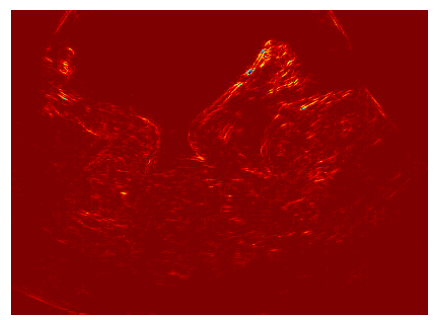

(d)

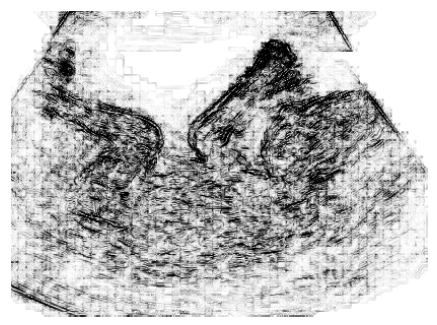

(f)

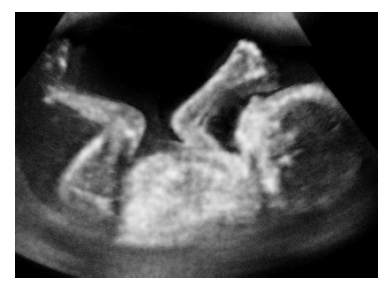

(g)

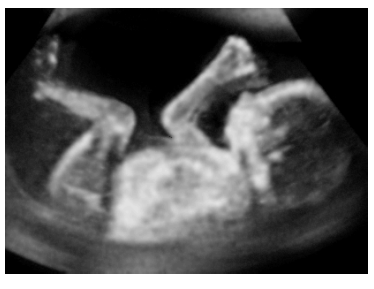

(h)

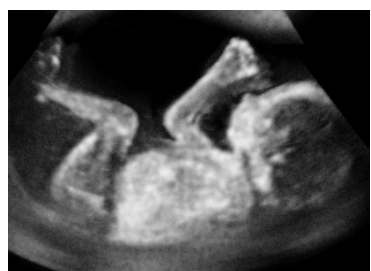

(i)

Figure 5: (a) Original image, (b) noisy one, (c) topological gradient for the second-order model, (d) topological gradient for the fourth-order model, (e) the variable exponent $q(\cdot)$, (f) the variable exponent $p(\cdot),(\mathrm{g}) p(\cdot)$ Kirchhoff model, (h) TVL model and (i) biharmonic model, $(\alpha=0.2$ and $\beta=0.2)$. 

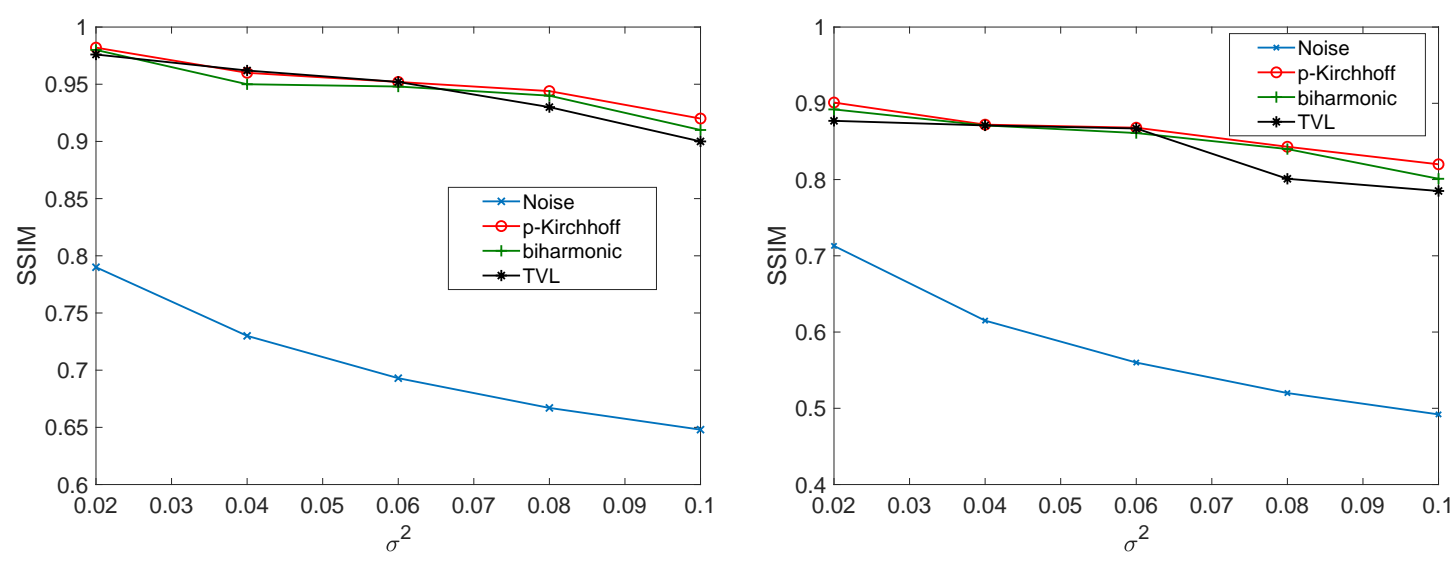

Figure 6: The sensitivity of noise level as a function of the SSIM for the three models applied to the images in Figures 4 and 5, respectively.

(a)

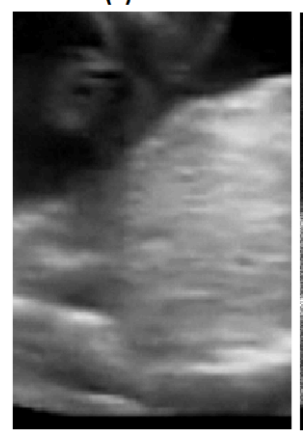

(b)

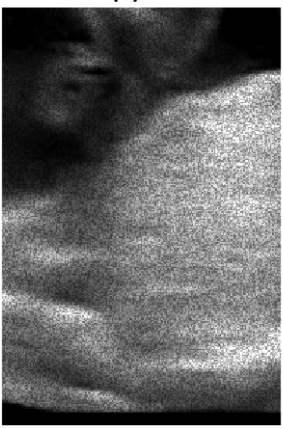

(c)

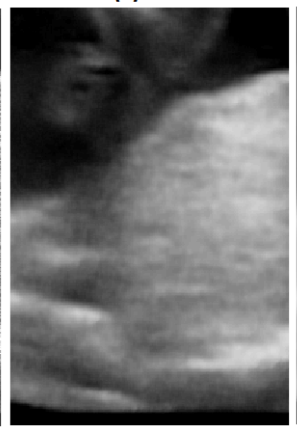

(d)

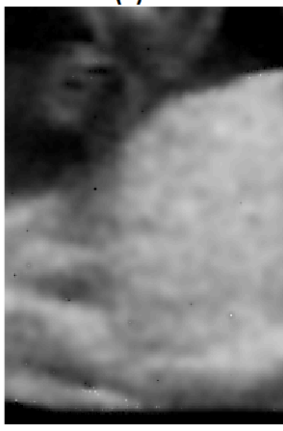

(e)

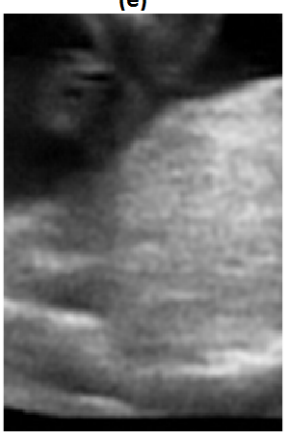

Figure 7: Zooms of Figure 4: (a) The original part, (b) the noisy one, (c) $p(\cdot)$-Kirchhoff model, (d) the TVL model and (e) the biharmonic model.

where $E$ is the fundamental solution of the $2 D$ bi-Laplace operator, which is given by

$$
E(x-y)=-\frac{1}{8 \pi}|x-y|^{2} \ln (|x-y|),
$$

and $\eta$ is the solution of the following boundary equations, for all $x \in \Sigma$, we have

$$
\begin{aligned}
& \oint_{\Sigma} \eta_{1}(y) \frac{\partial}{\partial n_{x}}\left(\frac{\partial \Delta E(x-y)}{\partial n_{y}}\right) \mathrm{d} \sigma(y)+\oint_{\Sigma} \eta_{2}(y) \frac{\partial\left(\Delta_{y} E(x-y)\right)}{\partial n_{x}} \mathrm{~d} \sigma(y)=g_{1}(x) \\
& \left.\oint_{\Sigma} \eta_{1}(y) \Delta_{x}\left(\frac{\partial(\Delta E(x-y))}{\partial n_{y}}\right) \mathrm{d} \sigma(y)+\oint_{\Sigma} \eta_{2}(y) \Delta_{x}\left(\Delta_{y} E(x-y)\right)\right) \mathrm{d} \sigma(y)=g_{2}(x)
\end{aligned}
$$

where $\oint$ denotes the Cauchy principal value. Moreover, the jump conditions across $\Sigma$ can be written as

$$
[H]=\eta_{1}, \quad\left[\partial_{n} H\right]=-\eta_{2} .
$$


If $\Sigma$ is a straight crack written as $\Sigma=(-1,1) \times\{0\}, g_{1}(x)=0$ and $g_{2}(x)=C$ st, then

$$
\eta_{1}(x)=0, \quad \eta_{2}(x)=\operatorname{Cst} \sqrt{1-x_{1}^{2}}, \quad \forall x=\left(x_{1}, 0\right) \in \Sigma .
$$

Proof. The well-posedness of problem (A.1) results from the Deny-Lions inequality [20]. The integral representation and the jump relation are obtained by using a similar method to that presented in $[6,18]$. The values of $\eta_{1}$ and $\eta_{2}$ for a straight crack have been obtained in [6].

\section{A.2 The topological gradient asymptotic}

Let $X_{\varepsilon}=\left\{u \in H^{2}\left(\Omega_{\varepsilon}\right)\left|\frac{\partial u}{\partial n}\right|_{\partial \Omega}=0\right\}, \Omega_{\varepsilon}=\Omega \backslash \overline{\left\{x_{0}+\varepsilon \sigma\right\}}$ and to simplify the presentation we assume that $x_{0} \equiv 0$. We set $\sigma_{\varepsilon}=\left\{x, \frac{x}{\varepsilon} \sigma\right\}$ and we assume that $\partial \Omega \cap \sigma_{\varepsilon}=\varnothing$.

The variational formulation of the perturbed equation (4.2) is:

$$
\text { Find } u_{\varepsilon} \in X_{\varepsilon} \text { such that, }\left\langle\mathcal{F}_{\varepsilon}\left(u_{\varepsilon}\right), \varphi\right\rangle=0, \quad \forall \varphi \in X_{\varepsilon} \text {, }
$$

where

$$
\left\langle\mathcal{F}_{\varepsilon}\left(u_{\varepsilon}\right), \varphi\right\rangle=\alpha \int_{\Omega_{\varepsilon}} \Delta u_{\varepsilon} \Delta \varphi \mathrm{d} x+\lambda \int_{\Omega_{\varepsilon}} D_{u} W\left(u_{\varepsilon}, f\right) \varphi \mathrm{d} x .
$$

Here, the brackets $\langle\cdot, \cdot\rangle$ denotes the duality product between $X_{\varepsilon}$ and $X_{\varepsilon}^{\prime}$. For brevity, in the sequel we write $\mathcal{F}^{v}(u):=\langle\mathcal{F}(u), v\rangle$.

We introduce the Lagrangian associated with problem (A.4) for all $\varphi, \phi \in X_{\varepsilon}$

$$
\mathcal{L}(\varphi, \phi):=\mathcal{J}_{\varepsilon}(\varphi)+\mathcal{F}_{\varepsilon}^{\phi}(\varphi) .
$$

The adjoint state equation is obtained by differentiating $\mathcal{L}$ with respect to $\varphi$ evaluated at $\varphi=u$ and $\phi=v_{\varepsilon}$,

$$
D_{\varphi} \mathcal{L}(\varphi, \phi)=0, \quad \forall \varphi \in X_{\varepsilon}
$$

or, equivalently

$$
D_{u} \mathcal{F}_{\varepsilon}^{v_{\varepsilon}}(u)(\varphi)=-D_{u} J_{\varepsilon}(u)(\varphi), \quad \forall \varphi \in X_{\varepsilon}
$$

where

$$
D_{u} \mathcal{F}_{\varepsilon}^{v_{\varepsilon}}(u)(\varphi)=\alpha \int_{\Omega_{\varepsilon}} \Delta v_{\varepsilon} \Delta \varphi \mathrm{d} x+\lambda \int_{\Omega_{\varepsilon}} D_{u}^{2} W(u, f) v_{\varepsilon} \varphi \mathrm{d} x .
$$

Using Green's formula, we deduce the corresponding strong form of (A.5)

$$
\begin{cases}\alpha \Delta^{2} v_{\varepsilon}+\lambda D_{u}^{2} W(u, f) v_{\varepsilon}=-\alpha \Delta^{2} u, & \text { in } \Omega_{\varepsilon}, \\ \frac{\partial v_{\varepsilon}}{\partial n}=\frac{\partial \Delta v_{\varepsilon}}{\partial n}=0, & \text { on } \partial \Omega, \\ \Delta v_{\varepsilon}=\frac{\partial \Delta v_{\varepsilon}}{\partial n}=0, & \text { on } \sigma_{\varepsilon} .\end{cases}
$$


Let $u_{\varepsilon}$ and $v_{\varepsilon}$ be the solutions of (4.2) and (A.5), respectively, and $u$ the solution of (3.6) with $p(x)=2$ and $\beta=0$. Taylor's expansion of $\mathcal{F}_{\varepsilon}$ and (A.5) gives

$$
\begin{aligned}
\mathcal{F}_{\varepsilon}^{v_{\varepsilon}}\left(u_{\varepsilon}\right)-\mathcal{F}_{\varepsilon}^{v_{\varepsilon}}(u) & =D_{u} \mathcal{F}_{\varepsilon}^{v_{\varepsilon}}(u)\left(u_{\varepsilon}-u\right)+\frac{1}{2} D_{u}^{2} \mathcal{F}_{\varepsilon}^{v_{\varepsilon}}\left(u_{\zeta_{\varepsilon}}\right)\left(u_{\varepsilon}-u\right)^{2} \\
& =-D_{u} J_{\varepsilon}(u)\left(u_{\varepsilon}-u\right)+E_{1}(\varepsilon)
\end{aligned}
$$

where

$$
\begin{aligned}
& E_{1}(\varepsilon)=\frac{1}{2} D_{u}^{2} \mathcal{F}_{\varepsilon}^{v_{\varepsilon}}\left(u_{\zeta_{\varepsilon}}\right)\left(u_{\varepsilon}-u\right)^{2}=\frac{1}{2} \int_{\Omega_{\varepsilon}} D_{u}^{3} W\left(u_{\zeta_{\varepsilon}} f\right)\left(u_{\varepsilon}-u\right)^{2} v_{\varepsilon} \mathrm{d} x, \\
& u_{\zeta_{\varepsilon}}=\theta u+(1-\theta) u_{\varepsilon}, \quad 0 \leq \theta \leq 1 .
\end{aligned}
$$

From Green's formula and (A.7), we get

$$
\begin{aligned}
J_{\varepsilon}\left(u_{\varepsilon}\right)-J_{0}(u) & =\alpha \int_{\Omega_{\varepsilon}}\left(\Delta u_{\varepsilon}-\Delta u\right) \Delta u \mathrm{~d} x+\frac{\alpha}{2} \int_{\Omega_{\varepsilon}}\left|\Delta u_{\varepsilon}-\Delta u\right|^{2} \mathrm{~d} x, \\
& =\mathcal{F}_{\varepsilon}^{v_{\varepsilon}}(u)-\mathcal{F}_{\varepsilon}^{v_{\varepsilon}}\left(u_{\varepsilon}\right)-E_{1}(\varepsilon)+E_{2}(\varepsilon)+E_{3}(\varepsilon)+E_{4}(\varepsilon),
\end{aligned}
$$

where

$$
\begin{aligned}
& E_{2}(\varepsilon)=\frac{\alpha}{2} \int_{\Omega_{\varepsilon}}\left|\Delta u_{\varepsilon}-\Delta u\right|^{2} \mathrm{~d} x, \\
& E_{3}(\varepsilon)=\alpha \int_{\sigma_{\varepsilon}} \frac{\partial \Delta u}{\partial n}\left[u_{\varepsilon}-u\right] \mathrm{d} \tau(x), \\
& E_{4}(\varepsilon)=-\alpha \int_{\sigma_{\varepsilon}} \Delta u\left[\frac{\partial\left(u_{\varepsilon}-u\right)}{\partial n}\right] \mathrm{d} \tau(x) .
\end{aligned}
$$

Then, using (A.4) and Green's formula on $\mathcal{F}_{\varepsilon}^{v_{\varepsilon}}(u)$ and we set $w_{\varepsilon}=v_{\varepsilon}-v_{0}$, where $v_{0}$ and $v_{\varepsilon}$ are solutions of (4.4) and (A.6), respectively. Thus, the variation of the cost function $J_{\varepsilon}\left(u_{\varepsilon}\right)-J_{0}(u)$ can be written as

$$
\begin{aligned}
J_{\varepsilon}\left(u_{\varepsilon}\right)-J_{0}(u)= & -\alpha \int_{\sigma_{\varepsilon}} \Delta u\left[\frac{\partial w_{\varepsilon}}{\partial n}\right] \mathrm{d} \tau(x)+\alpha \int_{\sigma_{\varepsilon}} \frac{\partial \Delta u}{\partial n}\left[w_{\varepsilon}\right] \mathrm{d} \tau(x)-E_{1}(\varepsilon) \\
& \quad+E_{2}(\varepsilon)+E_{3}(\varepsilon)+E_{4}(\varepsilon) \\
= & A-E_{1}(\varepsilon)+E_{2}(\varepsilon)+E_{3}(\varepsilon)+E_{4}(\varepsilon)+E_{5}(\varepsilon)
\end{aligned}
$$

where

$$
\begin{aligned}
& A=-\alpha \int_{\sigma_{\varepsilon}} \Delta u\left[\frac{\partial w_{\varepsilon}}{\partial n}\right] \mathrm{d} \tau(x), \\
& E_{5}(\varepsilon)=\alpha \int_{\sigma_{\varepsilon}} \frac{\partial \Delta u}{\partial n}\left[w_{\varepsilon}\right] \mathrm{d} \tau(x) .
\end{aligned}
$$


To compute the topological derivative of the cost function we need to approximate the solutions of the adjoint state $w_{\varepsilon}=v_{\varepsilon}-v_{0}$. We recall that $w_{\varepsilon} \in X_{\varepsilon}$ is solution of

$$
\begin{cases}\alpha \Delta^{2} w_{\varepsilon}+\lambda D_{u}^{2} W(u, f) w_{\varepsilon}=0, & \text { on } \Omega_{\varepsilon}, \\ \frac{\partial \Delta w_{\varepsilon}}{\partial n}=\frac{\partial w_{\varepsilon}}{\partial n}=0, & \text { in } \partial \Omega, \\ \frac{\partial \Delta w_{\varepsilon}}{\partial n}=-\frac{\partial \Delta v_{0}}{\partial n}, & \text { in } \sigma_{\varepsilon}, \\ \Delta w_{\varepsilon}=-\Delta v_{0}, & \text { in } \sigma_{\varepsilon} .\end{cases}
$$

We split $w_{\varepsilon}$ as

$$
w_{\varepsilon}=\varepsilon^{2} H\left(\frac{x}{\varepsilon}\right)+e_{\varepsilon}
$$

where $H \in W^{2}\left(\mathbb{R}^{2} \backslash \bar{\sigma}\right) / \mathbb{P}_{1}$ is the solution of the following boundary-value problem

$$
\begin{cases}\Delta^{2} H=0, & \text { in } \mathbb{R}^{2} \backslash \bar{\sigma}, \\ \frac{\partial \Delta H}{\partial n}=0, & \text { on } \sigma, \\ \Delta H=-\Delta v_{0}(0), & \text { on } \sigma,\end{cases}
$$

and $e_{\varepsilon}$ is the solution of the following equation

$$
\begin{cases}\alpha \Delta^{2} e_{\varepsilon}+\lambda D_{u}^{2} W(u, f) e_{\varepsilon}=-\varepsilon^{2} \lambda D_{u}^{2} W(u, f) H\left(\frac{x}{\varepsilon}\right), & \text { in } \Omega, \\ \frac{\partial \Delta e_{\varepsilon}}{\partial n}=-\frac{1}{\varepsilon} \frac{\partial H}{\partial n}\left(\frac{x}{\varepsilon}\right), & \text { on } \partial \Omega, \\ \frac{\partial \varepsilon_{\varepsilon}}{\partial n}=-\varepsilon \frac{\partial H}{\partial n}\left(\frac{x}{\varepsilon}\right), & \text { on } \partial \Omega, \\ \Delta e_{\varepsilon}=-\left(\Delta v_{0}-\Delta v_{0}(0)\right), & \text { on } \sigma_{\varepsilon}, \\ \frac{\partial \Delta e_{\varepsilon}}{\partial n}=-\frac{\partial \Delta v_{0}}{\partial n}, & \text { on } \sigma_{\varepsilon} .\end{cases}
$$

Using classical computations as it is done in [6], we have

$$
\left\|e_{\varepsilon}\right\|_{2, \Omega_{\varepsilon}}=O\left(-\varepsilon^{2} \log \varepsilon\right), \quad\left\|w_{\varepsilon}\right\|_{2, \Omega_{\varepsilon}}=O(\varepsilon) .
$$

We proceed now to evaluate the asymptotic expansion of the cost function (A.12). Using (A.15) the jump relation (??) and using the change of variable $x=\varepsilon y$, we obtain

$$
A=\alpha \varepsilon^{2} \Delta u(0) \int_{\sigma} \eta_{2}(y) \mathrm{d} \tau(y)+E_{6}(\varepsilon)+E_{7}(\varepsilon),
$$

where

$$
\begin{aligned}
& E_{6}(\varepsilon)=-\varepsilon \alpha \int_{\sigma}(\Delta u(\varepsilon y)-\Delta u(0))\left[\frac{\partial w_{\varepsilon}}{\partial n}(\varepsilon y)\right] d y, \\
& E_{7}(\varepsilon)=-\varepsilon \alpha \int_{\sigma} \Delta u(\varepsilon y)\left[\frac{\partial e_{\varepsilon}}{\partial n}(\varepsilon y)\right] d y .
\end{aligned}
$$


Using the asymptotic expansion of the density $\eta_{2}$ in the case of straight crack (A.3) with $g_{2}=-\Delta v_{0}(0)$ (see equation (A.1)), we obtain

$$
\begin{aligned}
A & =-\varepsilon^{2} \alpha \frac{4}{3} \Delta u(0) \Delta v_{0}(0) \int_{-1}^{1} \sqrt{1-y_{2}} \mathrm{~d} \tau\left(y_{2}\right)+E_{6}(\varepsilon)+E_{7}(\varepsilon) \\
& =-\varepsilon^{2} \alpha \frac{2 \pi}{3} \Delta u(0) \Delta v_{0}(0)+E_{6}(\varepsilon)+E_{7}(\varepsilon) .
\end{aligned}
$$

Standard computations similar that used in $[6,23]$ lead to

$$
E_{i}(\varepsilon)=o\left(\varepsilon^{2}\right), \quad \text { for } 1 \leq i \leq 7 .
$$

It follows from (A.12), (A.21) and (A.22) that the topological gradient at $x_{0}=0$ is given by

$$
G^{F}(0)=-\alpha \frac{2 \pi}{3} \Delta u(0) \Delta v_{0}(0) .
$$

For all points $x_{0}$ in $\Omega$ the general expression of the topological gradient for the fourthorder PDE of a cracked domain $\Omega \backslash\left\{x_{0}+\varepsilon \sigma(n)\right\}$ is given in (4.3).

\section{B Existence and uniqueness of a minimizer for energy (4.6)}

In this appendix, we establish the well-posedness of energy (4.6). Let us consider a minimizing sequence $\left(u_{n}\right)_{n} \subset \mathcal{H}^{p(\cdot)}(\Omega)$ of $\mathcal{E}(\cdot)$, then there exists $C>0$ such that

$$
\mathcal{E}\left(u_{n}\right) \leq C \text {. }
$$

Thus, $J^{p, q}\left(u_{n}\right) \leq C$ and hence $\left\|\Delta u_{n}\right\|_{L^{p(\cdot)}}$ and $\left\|\nabla u_{n}\right\|_{L^{q(\cdot)}}$ are uniformly bounded. Moreover, from the definition of the operator $\iota_{C}(\cdot)$ and the inequality (B.1), we necessary get that $\iota_{C}\left(u_{n}\right)=0$, which means that $u_{n} \in[m, M]$ and then $u_{n}$ is uniformly bounded in $L^{2}(\Omega)$. Therefore, using Proposition 2.1-a) and the fact that $1<p(\cdot), q(\cdot) \leq 2$, we get that $\left\|u_{n}\right\|_{L^{p(\cdot)}}$ and $\left\|u_{n}\right\|_{L q(\cdot)}$ are uniformly bounded. Thus, we obtain that $\left(u_{n}\right)_{n}$ is uniformly bounded in $\mathcal{H}^{p(\cdot)}(\Omega)$ which means that there exists a subsequence, still denoted $\left(u_{n}\right)_{n \in \mathbb{N}}$, such that $u_{n} \underset{n \rightarrow \infty}{\rightarrow} u$ weakly in $\mathcal{H}^{p(\cdot)}(\Omega)$ and the limit $u$ is a minimizer of $\mathcal{E}(\cdot)$. By using the lower semi-continuity of $\mathcal{E}(\cdot)$ and Fatou's Lemma we obtain that $u$ is a solution of (4.6). Uniqueness follows from the strict convexity of $\mathcal{E}(\cdot)$ for $u>0$.

\section{References}

[1] M. Allaoui. Existence of three solutions for variable exponent elliptic systems. Annali dell'Universiatà di Ferrara, 61(2):241-253, 2015.

[2] S. Amstutz. Topological sensitivity analysis for some nonlinear PDE system. J. Math. Pures Appl., 85(4):540-557, 2006.

[3] S. Amstutz and J. Fehrenbach. Edge detection using topological gradients: A scale-space approach. J. Math. Imaging Vis., 52(2):249-266, 2015. 
[4] S. Amstutz, I. Horchani and M. Masmoudi. Crack detection by the topological gradient method. Control Cybern., 34(1):81-101, 2005.

[5] S. Amstutz, A. A. Novotny and N. Van Goethem. Topological sensitivity analysis for elliptic differential operators of order 2m. J. Diff. Eq., 256(4):1735-1770, 2014.

[6] G. Aubert and A. Drogoul. Topological gradient for a fourth order operator used in image analysis. ESAIM-Control OPtim. Calc. Var., 21(4):1120-1149, 2015.

[7] D. Auroux. From restoration by topological gradient to medical image segmentation via an asymptotic expansion. Math. Comput. Model., 49(11):2191-2205, 2009.

[8] A. Beck and M. Teboulle. A fast iterative shrinkage-thresholding algorithm for linear inverse problems. SIAM J. Imaging Sci., 2(1):183-202, 2009.

[9] M. Belloni and B. Kawohl. A direct uniqueness proof for equations involving the $p$-Laplace operator. Manuscripta Mathematica, 109(2):229-231, 2002.

[10] Kh. Ben Haddouch, Z. El Allali, N. Tsouli and et al. Existence of solutions for a fourth order eigenvalue problem with variable exponent under Neumann boundary conditions. Bol. Soc. Paran. Mat., 34(1):253-272, 2016.

[11] M. Benning, C. Brune, M. Burger and et al. Higher-order TV methods enhancement via Bregman iteration. J. Sci. Comput., 54(2-3):269-310, 2013.

[12] E. Beretta, M.-C Cerutti, A. Manzoni and et al. An asymptotic formula for boundary potential perturbations in a semilinear elliptic equation related to cardiac electrophysiology. Math. Models Methods Appl. Sci., 26(4):645-670, 2016.

[13] K. Bredies, K. Kunisch and Th. Pock. Total generalized variation. SIAM J. Imaging Sci., 3(3):492-526, 2010.

[14] M. Burger, A. Sawatzky and G. Steidl. First order algorithms in variational image processing. In Splitting Methods in Communication, Imaging, Science, and Engineering, pages 345-407. Springer, 2016.

[15] J.-F. Cai, S. Osher and Z. Shen. Split Bregman methods and frame based image restoration. Multiscale Model. Simulation, 8(2):337-369, 2009.

[16] J. Céa, S. Garreau, Ph. Guillaume and et al. The shape and topological optimizations connection. Comput. Methods Appl. Mech. Engrg., 188(4):713-726, 2000.

[17] T. Chan, A. Marquina and P. Mulet. High-order total variation-based image restoration. SIAM J. Sci. Comput., 22(2):503-516, 2000.

[18] G. Chen and J. Zhou. Boundary element methods with applications to nonlinear problems. Atlantis Press, 2010.

[19] Y. Chen, S. Levine and M. Rao. Variable exponent, linear growth functionals in image restoration. SIAM J. Appl. Math., 66(4):1383-1406, 2006.

[20] J. Deny and J. L. Lions. Les espaces du type de Beppo-Levi. Ann. Inst. Fourier, 5:305-370, 1954.

[21] L. Diening, P. Harjulehto, P. Hästö and et al. Lebesgue and Sobolev Spaces with Variable Exponents. Springer, 2011.

[22] A. Drogoul. Numerical analysis of the topological gradient method for fourth order models and applications to the detection of fine structures in imaging. SIAM J. Imaging Sci., 7(4):2700-2731, 2016.

[23] A. Drogoul and G. Aubert. The topological gradient method for semi-linear problems and application to edge detection and noise removal. Inverse Probl. Imaging, 10(1):51-86, 2016.

[24] D. Edmunds and J. Rákosník. Sobolev embeddings with variable exponent. Studia Math., 143(3):267-293, 2000.

[25] D. E. Edmunds and J. Rákosník. Sobolev embeddings with variable exponent, II. Math. 
Nachr., 246(1):53-67, 2002.

[26] A. El Amrouss, F. Moradi and M. Moussaoui. Existence of solutions for fourth-order PDEs with variable exponents. Electron. J. Differ. Eq., 2009(153):1-13, 2009.

[27] X. Fan, J. Shen and D. Zhao. Sobolev embedding theorems for spaces $W^{k, p(x)}$. J. Math. Anal. Appl., 262(2):749-760, 2001.

[28] X. Fan and D. Zhao. On the spaces $L^{p(x)}$ and $W^{m, p(x)}$. J. Math. Anal. Appl., 263(2):424-446, 2001.

[29] R. Filippucci, P. Pucci, and F. Robert. On a $p$-Laplace equation with multiple critical nonlinearities. J. Math. Pures Appl., 91(2):156-177, 2009.

[30] S. Garreau, Ph. Guillaume, and M. Masmoudi. The topological asymptotic for PDE systems: The elasticity case. SIAM J. Control Optim., 39(6):1756-1778, 2000.

[31] P. Getreuer. Rudin-Osher-Fatemi total variation denoising using split Bregman. Image Processing On Line, 2:74-95, 2012.

[32] P. Getreuer. Total variation deconvolution using split Bregman. Image Processing On Line, 2:158-174, 2012.

[33] P. Getreuer. Total Variation inpainting using split Bregman. Image Processing On Line, 2:147157, 2012.

[34] A. Gillette. Image inpainting using a modified Cahn-Hilliard equation. PhD thesis, University of California, Los Angeles, 2006.

[35] T. Goldstein and S. Osher. The split Bregman method for L1-regularized problems. SIAM J. Imaging Sci., 2(2):323-343, 2009.

[36] P. Harjulehto, P. Hästö, V. Latvala and et al. Critical variable exponent functionals in image restoration. Appl. Math. Lett., 26(1):56-60, 2013.

[37] H. Houichet, M. Moakher and B. Rjaibi. Noise removal and edge detection in ultrasound images by the topological gradient method. Submitted to: New Trends in Mathematical Sciences, page 20 pages, 2018.

[38] M. Iguernane, S. A. Nazarov, J. R. Roche and et al. Topological derivatives for semilinear elliptic equations. Int. J. Appl. Math. Comput. Sci., 19(4-5):191-205, 2009.

[39] L. Jaafar Belaid, M. Jaoua, M. Masmoudi and et al. Application of the topological gradient to image restoration and edge detection. Enginee. Anal. Boundary Elements J., 32(11):891-899, 2008.

[40] Z. Jin and X. Yang. A variational model to remove the multiplicative noise in ultrasound images. J. Math. Imaging Vis., 39(1):62-74, 2011.

[41] K. Krissian, R. Kikinis, C. F. Westin, and K. Vosburgh. Speckle-constrained filtering of ultrasound images. In 2005 IEEE Computer Society Conference on Computer Vision and Pattern Recognition (CVPR'05), volume 2, pages 547-552, 2005.

[42] F. Li, Z. Li, and L. Pi. Variable exponent functionals in image restoration. Appl. Math. Comput., 216(3):870-882, 2010.

[43] P. Lindqvist. Notes on the p-Laplace Equation. University of Jyväskylä - Lectures notes, 2006.

[44] Th. Loupas. Digital image processing for noise reduction in medical ultrasonics. PhD thesis, University of Edinburgh, UK, 1988.

[45] D. L. Marks, T. S. Ralston, and S. A. Boppart. Speckle reduction by I-divergence regularization in optical coherence tomography. JOSA A, 22(11):2366-2371, 2005.

[46] M. Masmoudi. The topological asymptotic. In R. Glowinski, H. Kawarada, and J. Periaux, editors, Computational Methods for Control Applications, volume 16 of Math. Sci. Appl., pages 53-72. GAKUTO International, 2002.

[47] J. C. Nédélec. Acoustic and Electromagnetic Equations: Integral Representations for Harmonic 
Problems, volume 144. Springer Science \& Business Media, 2001.

[48] S. Osher, M. Burger, D. Goldfarb and et al. An iterative regularization method for total variation-based image restoration. Multiscale Modeling \& Simulation, 4(2):460-489, 2005.

[49] K. Papafitsoros, C. B. Schoenlieb and B. Sengul. Combined first and second order total variation inpainting using split Bregman. Image Processing On Line, 3:112-136, 2013.

[50] K. Papafitsoros and C. B. Schönlieb. A combined first and second order variational approach for image reconstruction. J. Math. Imaging . Vision, 48(2):308-338, 2014.

[51] M. Ruzicka. Electrorheological Fluids: Modeling and Mathematical Theory. Springer Science \& Business Media, 2000.

[52] S. Setzer. Split Bregman algorithm, Douglas-Rachford splitting and frame shrinkage. In International Conference on Scale Space and Variational Methods in Computer Vision, pages 464476. Springer, 2009.

[53] S. Setzer and G. Steidl. Variational methods with higher-order derivatives in image processing. In Approximation Theory XII: San Antonio 2007, 2008.

[54] B. Shi, Z.-F. Pang and J. Wu. Alternating split Bregman method for the bilaterally constrained image deblurring problem. Appl. Math. Comput., 250:402-414, 2015.

[55] J. Sokolowski and A. Zochowski. On the topological derivative in shape optimization. SIAM J. Control Optimizat., 37(4):1251-1272, 1999.

[56] C. Steger. Extracting curvilinear structures: A differential geometric approach. In European Conference on Computer Vision, pages 630-641. Springer, 1996.

[57] C. Steger. An unbiased detector of curvilinear structures. IEEE Transactions on Pattern Analysis and Machine Intelligence, 20(2):113-125, 1998.

[58] S. Wang, T.-Z. Huang, X.-L. Zhao, J.-J. Mei, and J. Huang. Speckle noise removal in ultrasound images by first-and second-order total variation. Numerical Algorithms, 78(2):513-533, 2018.

[59] Y. Wang, J. Yang, W. Yin, and Y. Zhang. A new alternating minimization algorithm for total variation image reconstruction. SIAM J. Imaging Sci., 1(3):248-272, 2008.

[60] C. Wu and X. C. Tai. Augmented Lagrangian method, dual methods, and split Bregman iteration for ROF, vectorial TV, and high order models. SIAM J. Imaging Sci., 3(3):300-339, 2010.

[61] V. V. Zhikov. Averaging of functionals of the calculus of variations and elasticity theory. Izvestiya: Mathematics, 29(1):33-66, 1987. 